\title{
Gallium Nitride Electrical Characteristics Extraction and Uniformity Sorting
}

\author{
Shyr-Long Jeng, ${ }^{1}$ Chih-Chiang Wu, ${ }^{2}$ and Wei-Hua Chieng ${ }^{2}$ \\ ${ }^{1}$ Department of Electrical and Electronic Engineering, Ta Hua University of Science and Technology, \\ No. 1, Dahua Road, Qionglin Shiang, Hsinchu County 30740, Taiwan \\ ${ }^{2}$ Department of Mechanical Engineering, National Chiao Tung University, No. 1001, University Road, Hsinchu City 30010, Taiwan
}

Correspondence should be addressed to Chih-Chiang Wu; wuchihchiang0517@gmail.com

Received 6 March 2015; Accepted 16 April 2015

Academic Editor: Meiyong Liao

Copyright (c) 2015 Shyr-Long Jeng et al. This is an open access article distributed under the Creative Commons Attribution License, which permits unrestricted use, distribution, and reproduction in any medium, provided the original work is properly cited.

\begin{abstract}
This study examined the output electrical characteristics-current-voltage $(I-V)$ output, threshold voltage, and parasitic capacitance-of novel gallium nitride $(\mathrm{GaN})$ power transistors. Experimental measurements revealed that both enhanced- and depletion-mode GaN field-effect transistors (FETs) containing different components of identical specifications yielded varied turnoff impedance; hence, the FET quality was inconsistent. Establishing standardized electrical measurements can provide necessary information for designers, and measuring transistor electrical characteristics establishes its equivalent-circuit model for circuit simulations. Moreover, high power output requires multiple parallel power transistors, and sorting the difference between similar electrical characteristics is critical in a power system. An isolated gate driver detection method is proposed for sorting the uniformity from the option of the turn-off characteristic. In addition, an equivalent-circuit model for GaN FETs is established on the basis of the measured electrical characteristics and verified experimentally.
\end{abstract}

\section{Introduction}

Metal-oxide-semiconductor field-effect transistors (MOSFETs) have been widely used over the past 30 years. As silicon approaches its performance limits, wide-bandgap semiconductors, such as gallium nitride $(\mathrm{GaN})$ and silicon carbide $(\mathrm{SiC})$, are emerging technologies that can supersede silicon MOSFETs as next-generation power transistors. Novel wide-band III-nitride semiconductor materials are being rapidly developed because of their unique properties, such as high electron mobility, saturation velocity, sheet carrier concentration at heterojunction interfaces, and breakdown voltages $[1,2]$. These properties make III-nitrides feasible for high-power, high-temperature applications. Compared with $\mathrm{SiC}, \mathrm{GaN}$ has low turn-on and switching losses and is less expensive. In addition, GaN wafers are produced by numerous manufacturers, thus negating any monopoly concerns. Furthermore, GaN has been widely used in light-emitting diodes and wireless applications. GaN power FETs are suitable for high-voltage, high-current, and motor-control applications as well as for industrial automation systems and automotive electronics [3-5].

Because both the commercially enhanced-mode (Emode) and depletion-mode (D-mode) GaN FETs manufactured by National Chiao Tung University (NCTU) $[6,7]$ are relatively new types of power transistors, few related studies are available in the literature. In addition, few manufacturers discuss them because commercial applications are not yet prevalent. The electrical characteristics of commercially manufactured power transistors differ because of the differences in cutting, wiring, wire bonding materials and diameters, and packaging. Before using such power transistors in circuit applications, their electrical characteristics must be extracted and sorted to match similar electrical properties in circuit designs. Unfortunately, extracting similar electrical properties is time-consuming and expensive. Thus, rapid and easy extraction of the electrical characteristics of GaN FETs to sort similar electrical properties is essential. 
Moreover, GaN FETs and the design of their gate drivers are relatively new. When using GaN FET power transistors in circuit applications, their unique electrical properties must be considered: (1) no intrinsic body diode [8-10], (2) low gateto-source voltage limits $[9,10]$, (3) full-conduction voltage of the gate and uncommon power supply voltage (e.g., $7 \mathrm{~V}$, $8 \mathrm{~V})[11-13]$, and (4) low threshold voltage $[9,10,12,13]$. The following properties are applicable to bridge-leg architecture power transistors: (1) floating source of the high-side power switch [14, 15] and (2) faulty turn-on [9, 15]. Therefore, GaN-FET-based power transistors require appropriate gate drive circuits and methods to prevent overload. Although numerous studies have examined these properties, none have focused on the turn-off characteristic.

This paper first reports the output electrical characteristics of novel GaN power transistors and standardized electrical measurements to provide necessary information for designers. Second, an isolated gate driver detection method is proposed for sorting. Finally, this paper presents a simple and accurate equivalent-circuit model of GaN FETs for circuit simulations, established on the basis of the measured electrical characteristics and verified experimentally.

\section{Materials and Methods}

2.1. Measurement of GaN Electrical Characteristics. On the basis of MOSFET and GaN-FET datasheets, the following characteristics were used in this study: (1) breakdown voltage $200 \mathrm{~V}$, rated current $9 \mathrm{~A}$, on-resistance $0.4 \Omega$, and E-mode MOSFET [16]; (2) breakdown voltage $500 \mathrm{~V}$, rated current $6 \mathrm{~A}$, on-resistance $0.5 \Omega$, and D-mode MOSFET [17]; (3) breakdown voltage $200 \mathrm{~V}$, rated current $12 \mathrm{~A}$, on-resistance $25 \mathrm{~m} \Omega$, and E-mode GaN FET [18]; and (4) D-mode GaN FET manufactured in the laboratory as testing devices. The electrical characteristics measured were $I_{D}-V_{D}$ characteristics, threshold voltage, and parasitic capacitance.

2.1.1. $I_{D^{-}} V_{D}$ Characteristic Curve. $I_{D}-V_{D}$ curve measurements detect the maximum output current of the power transistor when the gate voltage $V_{\mathrm{GS}}$ is applied as the fullconduction voltage, and the full-conduction on-state resistance is applied between the drain and source $\left(R_{\mathrm{DS}(\mathrm{ON})}\right)$. The characteristic curve reveals the linear and saturation regions of the circuit, where the device can operate properly. According to the test circuit in [19], the output characteristics are drain current $I_{D}$ versus drain-source voltage $V_{\mathrm{DS}}$ measured under different gate voltages $V_{\mathrm{GS}}$ ranging from the gate turnoff to full-on voltage at intervals of $1 \mathrm{~V}$. The $V_{\mathrm{GS}}$ range of the $\mathrm{E}$ and D-mode MOSFET is 0 to $12 \mathrm{~V}$ and -12 to $0 \mathrm{~V}$, respectively, the $V_{\mathrm{GS}}$ range of the E-mode GaN FET is 0 to $5 \mathrm{~V}$, and that of the D-mode GaN FET manufactured by NCTU is -5 to $0 \mathrm{~V}$. Drain voltage generally uses a pulse mode input, which prevents excessive heat that affects the output characteristics. According to the datasheets, test pulse properties for E- and D-mode MOSFETs and E-mode GaN-FETs are a pulse width of $300 \mu$ s and a duty cycle (duty) $\leqq 2 \%$ [16-18]. In this study, a test pulse width of $300 \mu \mathrm{s}$, pulse period of $300 \mathrm{~ms}$, and duty cycle of $0.1 \%$ were used to measure the $I_{D}-V_{D}$ curve characteristics of the four aforementioned power transistors.
2.1.2. Threshold Voltage. Threshold voltage $\left(V_{\mathrm{TH}}\right)$ is the minimum gate bias required to turn the device on and produce a drain current specified in the datasheet. In the threshold voltage measurement circuit for E-mode power transistors, the drain and gate terminals of the power transistors are shorted $\left(V_{\mathrm{DS}}=V_{\mathrm{GS}}\right)$ [16]. The voltage to the gate terminal is gradually increased from the turn-off voltage $V_{\text {off }}(0 \mathrm{~V})$ until the measured current equals the specified drain current. For $\mathrm{D}$-mode power transistors, the drain terminal is connected to a fixed DC voltage source. Similar to the test procedure for E-mode transistors, the voltage to the gate terminal of the $\mathrm{D}$-mode transistor is gradually increased from $V_{\text {off }}(-5 \mathrm{~V})$ and changes in its drain current $I_{D}$ are observed. As specified in the datasheet [17], a DC voltage of $25 \mathrm{~V}$ is applied to the drain terminal of D-mode MOSFETs. D-mode GaN FETs, which applying to drain terminal's DC voltage value refer to $I_{D}-V_{D}$ characteristic curve while the drain voltage attains the saturation region under the power transistor, are fully opened $\left(V_{\mathrm{GS}}=0 \mathrm{~V}\right)$. In this study, laboratorymanufactured D-mode GaN FET saturation voltage was set to the voltage measured from the $I_{D}-V_{D}$ characteristic curve. The conduction threshold current of E- and D-mode MOSFET is $250 \mu \mathrm{A}[16,17]$ and that of E-mode GaN-FET is $3 \mathrm{~mA}$ [18]. The laboratory-manufactured D-mode GaN FET has no datasheet for referencing its conduction threshold current. Therefore, to obtain the gate terminal input voltage $V_{\mathrm{GS}}$ at which the drain current $I_{D}$ increases instantaneously, the experimental measurements of the drain current $I_{D}$ are transformed logarithmically [20]. The $V_{\mathrm{GS}}$ thus obtained is considered the threshold voltage $V_{\mathrm{TH}}$.

2.1.3. Parasitic Capacitance. A power device analyzer/curve tracer [21] was used to measure the capacitance because it supports the measurement of the three nonlinear capacitances: $C_{\mathrm{GD}}, C_{\mathrm{DS}}$, and $C_{\mathrm{GS}}$. Figure 1 depicts the $C_{\mathrm{GD}}, C_{\mathrm{DS}}$, and $C_{\mathrm{GS}}$ measurement circuits, respectively. A multiple frequency capacitance measurement unit with four ports $(\mathrm{Hp}$, $\mathrm{Hc}$, Lp, and Lc) was used for capacitance measurements. Hp and $\mathrm{Hc}$ were shorted together (hereafter, $\mathrm{CMH}$ ), and Lp and Lc were shorted together (hereafter, CML). The CMH outputs an AC test signal through the circuit under test, which is detected by the CML. The CMH operates in the $100 \mathrm{kHz}-$ $1 \mathrm{MHz}$ AC frequency range. As specified in the MOSFET and E-mode GaN-FET datasheets, $C_{\mathrm{GS}}$ is measured at $100 \mathrm{kHz}$, and $C_{\mathrm{DS}}$ and $C_{\mathrm{GS}}$ are measured at $1 \mathrm{MHz}$; the oscillation level is $30 \mathrm{mV}$ for all measurements. The CML receiving port potential is equivalent to the ground terminal.

The parasitic capacitance $C_{\mathrm{GD}}$ is measured using the circuit shown in Figure 1(a). The AC test signal is output from the $\mathrm{CMH}$ to the drain terminal, the source terminal is connected to a high-voltage source/monitor unit (HVSMU), and the source terminal grounds the AC signal to the AC guard. Therefore, the AC test signal from $C_{\mathrm{DS}}$ to the source terminal is grounded by the AC guard, preventing the signals from being received by the source terminal through the $C_{\mathrm{GS}}$ path. When measuring the parasitic capacitance $C_{\mathrm{GD}}$, the HVSMU should provide a $V_{\text {off }}$ DC bias relative to the CML gate terminal, and the power transistor should be kept off. Both the enhanced MOSFET and E-mode GaN-FET power 


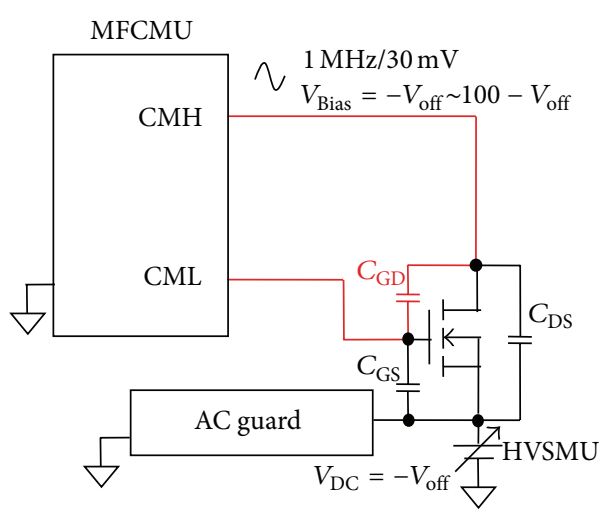

(a)

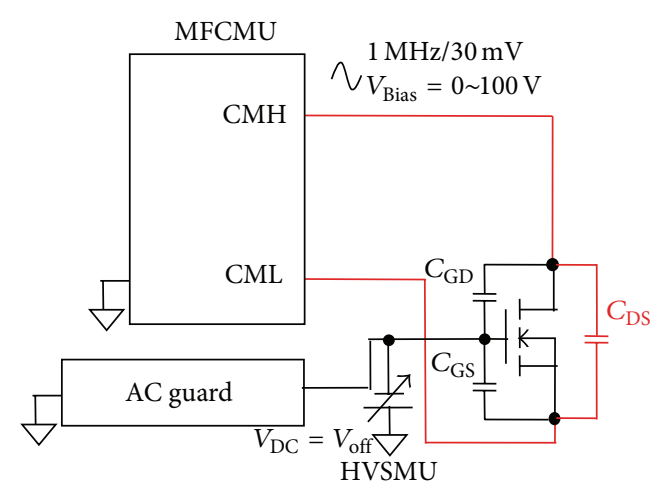

(b)

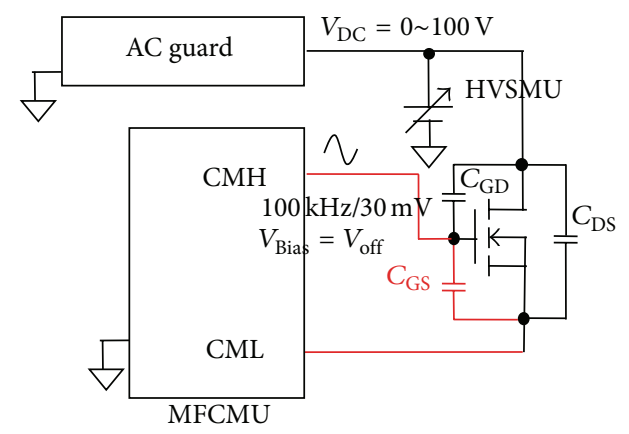

(c)

FIgure 1: Test circuit for (a) $C_{\mathrm{GD}}$ capacitance, (b) $C_{\mathrm{DS}}$ capacitance, and (c) $C_{\mathrm{GS}}$ capacitance.

transistor have the same $V_{\text {off }}(0 \mathrm{~V})$, whereas that of the Dmode MOSFET power transistor is $-12 \mathrm{~V}$. However, the $I_{D}-V_{D}$ curve revealed that the tested D-mode MOSFET turns off at $-5 \mathrm{~V}$. Therefore, in this study, $-5 \mathrm{~V}$ was used as the $V_{\text {off }}$ for the $D$-mode MOSFET power transistor. The $V_{\text {off }}$ of D-mode GaN FET is $-5 \mathrm{~V}$. Because the source terminal has a bias voltage, $-V_{\text {off }}$, the CMH should apply an AC bias voltage in the range $V_{\text {off }}$ to $100-V_{\text {off }}$ to drain the terminal. Therefore, the bias $V_{\mathrm{DS}}$ voltage of measurement can range from 0 to $100 \mathrm{~V}$.

The parasitic capacitance $C_{\mathrm{DS}}$ is measured using the circuit shown in Figure 1(b). The circuit is similar to that used to measure $C_{\mathrm{GD}}$; the only difference is that the gate terminal was changed to the source terminal to connect the CML. The $\mathrm{CMH}$ outputs an AC test signal to the drain terminal and receives an $\mathrm{AC}$ test signal from the CML by connecting CML to the source of the device under test. The gate terminal grounds the AC guard to prevent the $\mathrm{AC}$ test signals from being received by the source terminal through the $C_{\mathrm{GD}}$ and $C_{\mathrm{GS}}$ paths. To ensure that the power transistor remains off during the measurement, the DC voltage $V_{\text {off }}$ is applied to the gate terminal. The $\mathrm{CMH}$ gradually increases the $\mathrm{AC}$ bias voltage from 0 to $100 \mathrm{~V}$; therefore, the parasitic capacitance measurement $C_{\mathrm{DS}}$ is in the $V_{\mathrm{DS}}$ voltage range of $0-100 \mathrm{~V}$.

The parasitic capacitance $C_{\mathrm{GS}}$ is measured using the circuit shown in Figure 1(c). The $\mathrm{CMH}$ outputs an AC test signal to the gate terminal, and the CML receives the signal by connecting to the source terminal. Because the $\mathrm{CMH}$ has a $V_{\text {off }}$ bias, the power transistor is kept off. To prevent the signals from being received by the source terminal through the $C_{\mathrm{GD}}$ and $C_{\mathrm{DS}}$ paths, the drain terminal is grounded to the AC guard. The HVSMU provides DC voltage in the range of $0-100 \mathrm{~V}$; therefore, parasitic capacitance $C_{\mathrm{GS}}$ is measured at different $V_{\mathrm{DS}}$ voltages in the range of $0-100 \mathrm{~V}$.

\subsection{Mechanism of Isolated Gate Drive Detection}

2.2.1. Conventional Gate Drive. Conventional power transistor gate drives use gate drive integrated circuit (IC) architecture. When used in half- and full-bridge-leg drive topologies, gate drives typically use an optical coupling IC to form an isolated floating-supply gate drive circuit architecture. Isolated gate drive circuit architecture consists of fast optical coupling IC, gate driver IC, and auxiliary supply voltage, as shown in Figure 2. The gate drive voltage for the E-mode GaN FET gate-to-source voltage is $V_{\mathrm{ISO}}-G_{\mathrm{ISO}}$, and the $V_{\mathrm{GS}}$ for $\mathrm{D}$-mode GaN FET gate drive voltage is $-V_{\text {ISO }}$ to $G_{\text {ISO }}$. The difference between the gate drive circuits for $\mathrm{E}$ - and D-mode GaN FETs is that the input supply voltages for the isolated gate driver amplifier are $\left(+V_{\text {ISO }}\right)-\left(G_{\text {ISO }}\right)$ and $\left(G_{\text {ISO }}\right)-\left(-V_{\text {ISO }}\right)$, respectively. The external gate drive signals of the isolated gate drive circuit for the E-mode GaN FET is $\left[0\right.$ to $\left.+V_{\text {ISO }}\right]$ relative to the ground (Gnd). Through the fast optical coupling IC, the signal is isolated and converted to $\left[0\right.$ to $\left.+V_{\text {ISO }}\right]$ relative to $G_{\text {ISO }}$. Finally, the isolated signal is amplified through the gate 


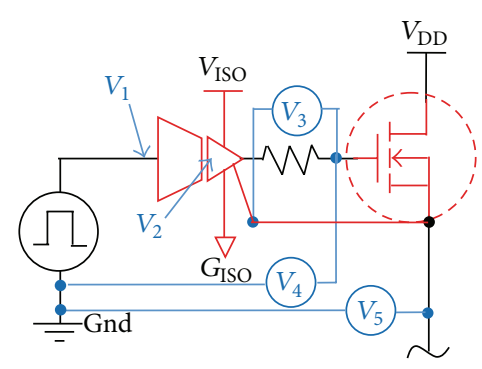

FIGURE 2: Isolated gate drive detection circuit.

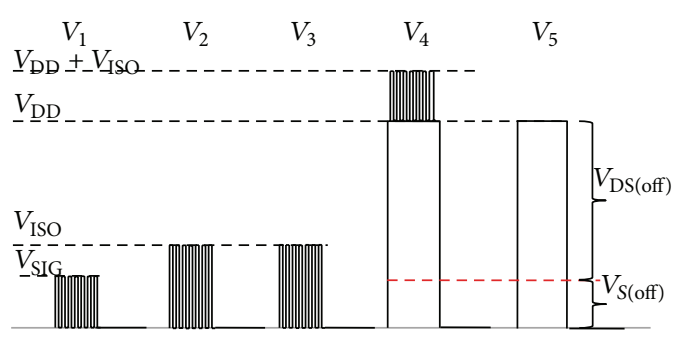

FIGURE 3: Detection drive signal for E-mode transistor. driver IC to drive the E-mode GaN FET. Similarly, the gate drive circuit for D-mode GaN FET is isolated to produce the gate signal $\left[-V_{\text {ISO }}-G_{\text {ISO }}\right]$ relative to $G_{\text {ISO }}$ to drive the FET.

2.2.2. Isolated Gate Drive Detection. The isolated gate drive signal waveform can be measured by an oscilloscope to distinguish the waveforms of the turn-off impedance $R_{\mathrm{DS} \text { (off) }}$ when GaN FETs are in the turn-off state. The proposed detection method is a relatively simple screening method to sort similar electrical characteristics of GaN FETs; by observing the switching waveforms, external stray capacitance in the circuit boards and internal parasitic capacitance in the transistors can be detected. The simple and accurate GaN FET model established on the basis of the measured electrical characteristics can be verified through experimental measurements of the isolated gate drive signal waveforms.

The proposed isolated gate drive detection circuit is illustrated in Figure 2, and the drive signal for the E-mode GaN FET is shown in Figure 3. Voltages $V_{1}, V_{4}$, and $V_{5}$ were measured relative to the Gnd; $V_{2}$ and $V_{3}$ were measured relative to the isolated supply ground $G_{\text {ISO }}$. The drain terminal of the E-mode GaN FET test circuit connects to the power supply voltage $V_{\mathrm{DD}}$ relative to the Gnd. When the gate-tosource voltage of the E-mode GaN FET is $V_{\text {ISO }}$, it turns on and shorts the drain and source terminals. Ideally, the source terminal voltage relative to the Gnd should be promoted to $V_{\mathrm{DD}}$. The source terminal of the GaN FET and the isolated power source terminal $G_{\text {ISO }}$ are connected, indirectly causing $G_{\text {ISO }}$ and GaN FET Gnd to turn on and off; therefore, $G_{\text {ISO }}$ has a relative floating voltage of $V_{\mathrm{DD}}$. When the gate terminal voltage of the GaN FET relative to the source terminal voltage is $V_{\text {ISO }}$, the E-mode GaN FET turns on, the isolated power supply ground $G_{\mathrm{ISO}}$ relative to the Gnd is $V_{\mathrm{DD}}$, and the voltage between the GaN FET gate terminal and the Gnd is $V_{\mathrm{DD}}+V_{\mathrm{ISO}}$. When the gate terminal voltage relative to the source terminal is $0 \mathrm{~V}$, the E-mode GaN FET turns off, the gate and source terminals are open, and $G_{\text {ISO }}$ and Gnd are 0. Because the GaN FET turns off, the drain terminal voltage $V_{\mathrm{DD}}$ no longer offers voltage to $G_{\mathrm{ISO}}$. The floating voltage $V_{\mathrm{DD}}$ discharges through circuit stray capacitance $C_{\text {stray }}$ and load resistance $R_{L}$. Therefore, $G_{\text {ISO }}$ floating voltage discharges from $V_{\mathrm{DD}}$ at the speed of the resistor-capacitor time constant until the next pulse width modulation (PWM) signal to the gate-tosource voltage is $V_{\text {ISO }}$, which turns the GaN FET on again. When the drain terminal voltage $V_{\mathrm{DD}}$ is supplied to $G_{\mathrm{ISO}}$, the parasitic capacitance $C_{\text {ISS }}$ can be recharged to $V_{\mathrm{DD}}+V_{\mathrm{ISO}}$. The higher the PWM drive signal frequency entering the gate terminal, the more stable the $G_{\mathrm{ISO}}$ in maintaining $V_{\mathrm{DD}}$. When the PWM signal is no longer sent to the gate terminal and the GaN FET remains off for a sufficient period, $V_{\mathrm{DD}}$ discharges to $0 \mathrm{~V}$ through circuit stray capacitance $C_{\text {stray }}[22]$ and load resistance $R_{L}$, as shown in Figure $2\left(V_{5}\right)$. Concurrently, Gnd drops to $0 \mathrm{~V}$. Compared with the turn-off impedance of $\mathrm{Si}$ MOSFETs, the turn-off impedance $R_{\mathrm{DS} \text { (off) }}$ of the GaN FET is low. Therefore, the leakage current offers a load resistance $R_{L}$ to produce voltage $V_{S \text { (off) }}$. The turn-off impedance $R_{\mathrm{DS} \text { (off) }}$ of the GaN FET can be obtained by observing the $V_{S \text { (off) }}$ variance.

The D-mode GaN FET signal is depicted in Figure 4. When the gate-to-source terminal voltage of the D-mode GaN FET is $0 \mathrm{~V}$, because it is typically turned on, its drain and source terminals are short. In this case, the source-to-ground Gnd voltage should be increased to the power supply voltage $V_{\mathrm{DD}}$, and the GaN FET gate-to-ground Gnd voltage should be $V_{\mathrm{DD}}$. When the gate-to-source terminal voltage is $-V_{\mathrm{ISO}}$, the transistor turns off. Concurrently, the gate-to-ground Gnd becomes $V_{\mathrm{DD}}-V_{\mathrm{ISO}}$. The $G_{\mathrm{ISO}}$ voltage $V_{\mathrm{DD}}$ discharges through the circuit stray capacitance $C_{\text {stray }}[22]$ and the load resistance $R_{L}$, as shown in Figure $2\left(V_{5}\right)$, until the next PWM to the gateto-source terminal voltage is $0 \mathrm{~V}$, which turns the GaN FET on again. The drain terminal voltage $V_{\mathrm{DD}}$ provides voltage to $G_{\text {ISO }}$. When the gate terminal PWM signal ceases and the GaN FET remains off for a sufficient period, the gateto-ground Gnd voltage discharges through the circuit stray capacitance and resistance to $0 \mathrm{~V}$. The condition of the $\mathrm{D}$ mode GaN FET is the same as that of the E-mode GaN FET. The turn-off impedance $R_{\mathrm{DS} \text { (off) }}$ of D-mode GaN FET is smaller than that of Si MOSFET; therefore, the leakage current offers a load resistance $R_{L}$ to produce voltage $V_{S \text { (off) }}$. The relationship between the turn-off impedance $R_{\mathrm{DS} \text { (off) }}$, leakage current, and voltage $V_{S(\mathrm{off})}$ is discussed later.

2.2.3. Isolated Gate Driver Circuit Model. To make the device model adaptable to and suitable for a system-level simulation, a subcircuit model was developed using the measured characterization results as the parameters of the model [23]. A simplified isolated gate driver detection circuit architecture is shown in Figure 5(a). A voltage probe is used to measure the voltage between the gate terminal and the ground. When $V_{\mathrm{GS}}$ is $0 \mathrm{~V}$ (to turn the E-mode GaN FET off) or $-V_{\text {ISO }}$ (to turn the D-mode GaN FET off), the E- or D-mode GaN FETs are equivalent to a turn-off resistance $R_{\mathrm{DS} \text { (off) }}$, which can be used 


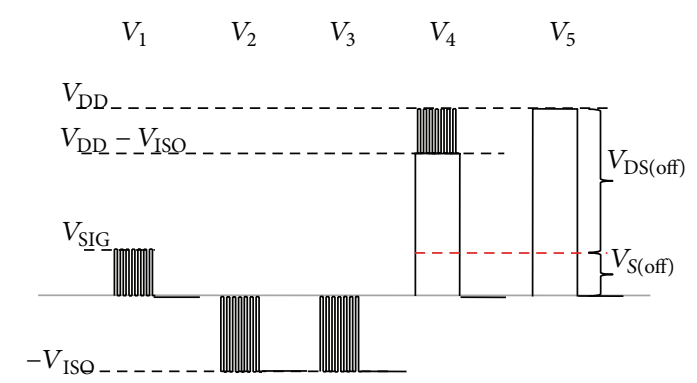

FIGURE 4: Detection drive signal for D-mode transistor.

to evaluate the turn-off capacity of the GaN FET. In this test, with a known probe resistance value $R_{L}$ and by applying the Kirchhoff laws, the current through the power supply voltage $V_{\mathrm{DD}}$ at resistances $R_{\mathrm{DS}(\text { off) }}$ and $R_{L}$ is obtained as the leakage current $I_{\mathrm{DSS}}$, which can be described as follows:

$$
I_{\mathrm{DSS}}=\frac{V_{\mathrm{DD}}}{R_{\mathrm{DS}(\mathrm{off})}+R_{L}} .
$$

The leakage current $I_{\text {DSS }}$ flows through the voltage probe and produces $V_{S(\text { off })}$ as follows:

$$
V_{S(\text { off })}=R_{L} I_{\mathrm{DSS}} .
$$

By substituting (1a) into (lb), the $R_{\mathrm{DS}(\text { off })}$ impedance can be derived as follows:

$$
R_{\mathrm{DS}(\mathrm{off})}=R_{L} \frac{V_{\mathrm{DD}}}{V_{S(\mathrm{off})}}-R_{L} .
$$

A simplified simulation of the isolated gate drive detection circuit is shown in Figure 5(b). The simplified simulation circuit consists of the controlled signal source, E- or D-mode GaN FET current source, the isolated power supply ground $G_{\mathrm{ISO}}$, ground $\mathrm{Gnd}$, the parasitic capacitances $C_{\mathrm{GS}}, C_{\mathrm{GD}}$, and $C_{\mathrm{DS}}$, turn-off impedance $R_{\mathrm{DS} \text { (off), }}$, and voltage probe resistance $R_{L}$. The turn-off resistance $R_{\mathrm{DS}(\text { off) }}$ and voltage probe resistance $R_{L}$ connect together and, with the applied voltage $V_{\mathrm{DD}}$ and ground Gnd, form a loop that can use the Kirchhoff voltage law to estimate the leakage current $I_{\mathrm{DSs}}$. The $I_{\mathrm{DS}}$ current source is extracted from the $I_{D}-V_{D}$ characteristics. The $I_{D}-V_{D}$ characteristic curve of the GaN FET follows the Level 1 MOSFET model characterized by (3a), (3b), and (3c) and is divided into cut-off (3a), linear (3b), and saturation (3c) regions.

Cut-off region:

$$
I_{\mathrm{DS}}=0 .
$$

Linear region:

$$
I_{\mathrm{DS}}=K_{P} \times\left[\left(V_{\mathrm{GS}}-V_{\mathrm{TH}}\right) \times V_{\mathrm{DS}}-\frac{1}{2} V_{\mathrm{DS}}^{2}\right] .
$$

Saturation region:

$$
I_{\mathrm{DS}}=\frac{1}{2} \times K_{P} \times\left(V_{\mathrm{GS}}-V_{\mathrm{TH}}\right)^{2} \times\left(1+\lambda \times V_{\mathrm{DS}}\right),
$$

where $K_{P}$ is the transduced value and $\lambda$ is the short-channel width-modulation slope coefficient in the saturated region, which is initially set to 0 . The sign of $V_{\mathrm{TH}}$ determines the mode: positive is for E-mode and negative is for D-mode. Using the established Level 1 MOSFET $I_{D}-V_{D}$ characteristics model equations to describe the GaN FET current value in the saturation region reveals a large difference between experimentally measured and simulated data. Therefore, referring to a smoothing equation, the coefficient $1 / 2$ in (3b) is replaced with $1 / 3$ and that in (3c) is replaced with $2 / 3$. The smoothing equation (4) is used to smoothen the $I_{D}-V_{D}$ characteristic curve in the linear and saturation regions; the $V_{\mathrm{GS}}-V_{\mathrm{TH}}$ voltage value is modified to $V_{\mathrm{GS} \text { _eff }}$ and substituted in (5a) and (5b). The + and - signs denote the D- and E-modes of the GaN FET, respectively, which complies with the $I_{D}-V_{D}$ characteristics of the GaN FET model. The $\delta$ value impacts the degree of smoothness between the linear and saturated regions; the higher the value is, the smoother the curve is [24]. The smoothing equation is as follows:

$$
\begin{gathered}
V_{\mathrm{GS} \_ \text {eff }}=\left(V_{\mathrm{GS}}-V_{\mathrm{TH}}\right)-\left(\frac{1}{3}\right)\left(\left(V_{\mathrm{GS}}-V_{\mathrm{TH}}\right)-V_{\mathrm{DS}}-\delta\right. \\
\left.+\sqrt{\left(\left(V_{\mathrm{GS}}-V_{\mathrm{TH}}\right)-V_{\mathrm{DS}}-\delta\right)^{2} \pm 4 \delta\left(V_{\mathrm{GS}}-V_{\mathrm{TH}}\right)}\right) .
\end{gathered}
$$

After smoothening, the GaN FET $I_{D}-V_{D}$ characteristic equations for the linear and saturation regions are depicted as follows:

$$
\begin{aligned}
& \text { Linear region }\left(V_{\mathrm{DS}} \leq V_{\mathrm{GS} \_ \text {eff }}\right) \text { : } \\
& \qquad I_{\mathrm{DS}}=K_{P} \times\left[V_{\mathrm{GS}_{-} \text {eff }} \times V_{\mathrm{DS}}-\frac{1}{3} V_{\mathrm{DS}}^{2}\right] .
\end{aligned}
$$

Saturation region $\left(V_{\mathrm{DS}}>V_{\mathrm{GS} \_ \text {eff }}\right)$ :

$$
I_{\mathrm{DS}}=\frac{2}{3} \times K_{P} \times V_{\mathrm{GS} \_ \text {eff }}^{2} .
$$

SPICE simulation software [25] was used to simulate the electrical characteristics and to verify the measured gate drive signals. The Shenai model [23] was used and the builtin Level 1 MOSFET capacitance model was replaced with external capacitances. The measured gate-source capacitance was relatively independent of $V_{\mathrm{DS}}$ voltage, and a constant measured capacitance $C_{\mathrm{GS}}$ was used in the circuit model. The $C_{\mathrm{GD}}$ and $C_{\mathrm{DS}}$ can be described using the following equations:

$$
\begin{aligned}
C_{\mathrm{GD}} & =\frac{C_{\mathrm{GD} 0}}{\left(1-V_{\mathrm{GD}} / V_{J}\right)^{m}}, \\
C_{\mathrm{DS}} & =\frac{C_{\mathrm{DS} 0}}{\left(1-V_{\mathrm{DS}} / V_{J}\right)^{m}},
\end{aligned}
$$

where $C_{\mathrm{GD} 0}$ is the zero-bias gate-to-drain capacitance, $C_{\mathrm{DS} 0}$ is the zero-bias drain-to-source capacitance, $V_{\mathrm{GD}}$ is the gateto-drain voltage, $V_{\mathrm{DS}}$ is the drain-to-source voltage, $V_{J}$ is the junction built-in potential, and $m$ is the junction grading coefficient. The parameters $V_{J}$ and $m$ were adjusted to obtain the optimal fit with the measured capacitance data. Moreover, the effect of external couple capacitances on $V_{\mathrm{GS}}$ during turnoff is considered. 


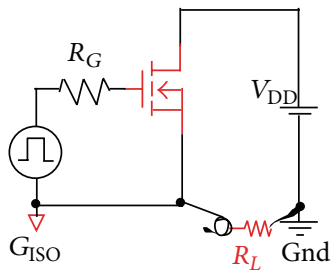

(a)

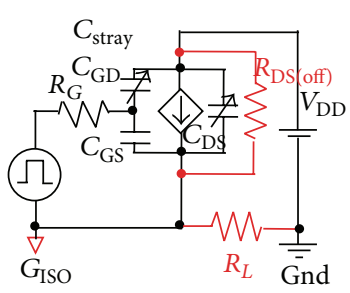

(b)

FIGURE 5: Simplified schematic of gate drive detecting circuit: (a) simplified schematic and (b) simplified equivalent model.

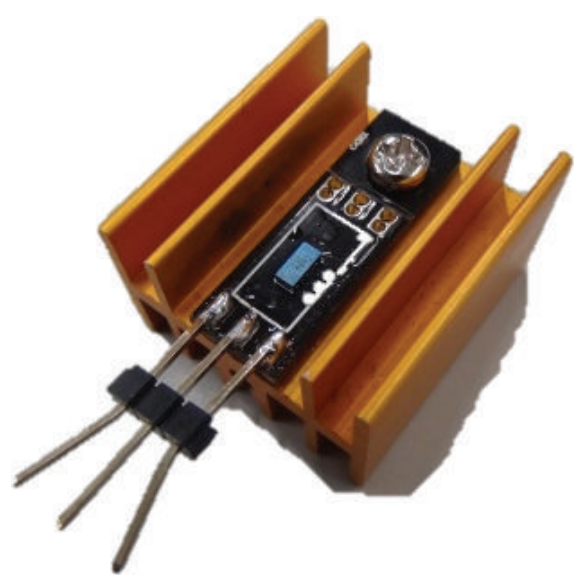

(a)

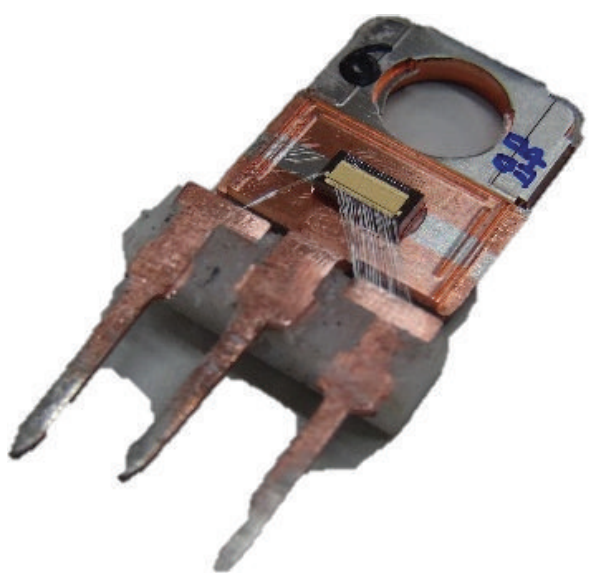

(b)

FIgUre 6: GaN FETs device under test: (a) E-mode GaN FET and (b) D-mode GaN FET.

\section{Results and Discussion}

3.1. $I_{D}-V_{D}$ Characteristic Curve. E- and D-mode GaN FET devices under test are shown in Figure 6. The tested Dmode GaN FET chip is $80 \mathrm{~mm}$ in size and is packaged in the TO-3P form. Figure 7 depicts the measured $I_{D}-V_{D}$ characteristics of the four power transistors. Solid lines represent the waveforms specified in the datasheets, dotted lines represent the measured waveforms, and solid lines with circles represent the SPICE-simulated waveforms.

The measured $I_{D}-V_{D}$ characteristics of the E- and Dmode MOSFET waveforms are similar to those specified in the datasheet. The on-resistance $R_{\mathrm{DS}(\mathrm{ON})}$ at a specific turnon gate voltage $V_{\mathrm{GS}}$ and drain current $I_{\mathrm{DS}}$ can be extracted directly from the output characteristic curves.

Figure 8 plots the $R_{\mathrm{DS}(\mathrm{ON})}$ of the four power transistors. In the enhanced MOSFET, $R_{\mathrm{DS}(\mathrm{ON})}$ at $V_{\mathrm{GS}}=10 \mathrm{~V}, V_{\mathrm{DS}}=1.44 \mathrm{~V}$, and $I_{\mathrm{DS}}=4.5 \mathrm{~A}$ is approximately $1.44 / 4.5=0.32 \Omega$, which is under the maximum value specified in the datasheet $(0.4 \Omega)$. In the D-mode MOSFET, $R_{\mathrm{DS}(\mathrm{ON})}$ at $V_{\mathrm{GS}}=0 \mathrm{~V}, V_{\mathrm{DS}}=1.66 \mathrm{~V}$, and $I_{\mathrm{DS}}=3 \mathrm{~A}$ is approximately $1.66 / 3=0.55 \Omega$, which is close to the value specified in the datasheet $(0.5 \Omega)$. Although the D-mode MOSFET $V_{\mathrm{GS}}$ is $0 \mathrm{~V}$, it conducts current but not at full conduction. When $V_{\mathrm{GS}}$ is $5 \mathrm{~V}, V_{\mathrm{DS}}$ is $30 \mathrm{~V}$ and the output current $I_{D}$ is $35 \mathrm{~A}$. When $V_{\mathrm{GS}}$ is $-2 \mathrm{~V}$, the power transistor turns off and the output current $I_{D}$ is close to zero. GaN FET output characteristic variation is considerably large compared with that of the MOSFET. The output current value exhibits drift phenomena in different E-mode GaN FET samples when the inputs $V_{\mathrm{GS}}$ and $V_{\mathrm{DS}}$ are the same. The experimental results show that the linear region of the onresistance $R_{\mathrm{ON}}$ is approximately $0.025-0.03 \Omega$. When $V_{\mathrm{GS}}$ is $5 \mathrm{~V}$ and the average output current $I_{D}$ is $6 \mathrm{~A}$, the average voltage $V_{\mathrm{DS}}$ is $0.18 \mathrm{~V}$; therefore, the average on-state resistance $R_{\mathrm{ON}}$ is $0.18 / 6=0.03 \Omega$, which exceeds the datasheet value of $0.025 \Omega$. The D-mode GaN FET on-resistance $R_{\mathrm{ON}}$ is approximately $0.25-0.30 \Omega$. When $V_{\mathrm{GS}}$ is $0 \mathrm{~V}$ and $V_{\mathrm{DS}}$ is $1 \mathrm{~V}$, $I_{D}$ is $3.87 \mathrm{~A}$; therefore, $R_{\mathrm{ON}}$ is $1 / 3.87=0.26 \Omega$.

Equations (5a) and (5b) are used to establish the current source model of a transistor. The output voltage $V_{\mathrm{DS}}$ of the E-mode GaN FET is increased from 0 to $3 \mathrm{~V}$ at intervals of $0.1 \mathrm{~V}$, and the gate input voltage is increased from 0 to $5 \mathrm{~V}$ at intervals of $1 \mathrm{~V}$; the output voltage $V_{\mathrm{DS}}$ of the $\mathrm{D}$-mode $\mathrm{GaN}$ FET is increased from 0 to $10 \mathrm{~V}$ at intervals of $0.5 \mathrm{~V}$, and the gate voltage is increased from -5 to $0 \mathrm{~V}$ at intervals of $1 \mathrm{~V}$.

The waveforms are shown in Figures 7(c) and 7(d) as solid lines with circles; the simulated characteristics are similar to the measured characteristics (dashed lines). Drain current 


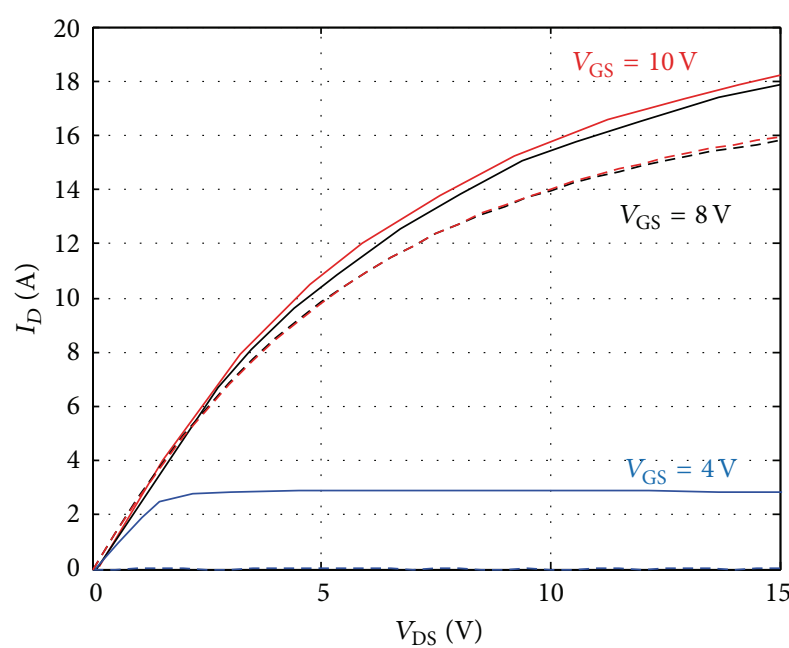

(a)

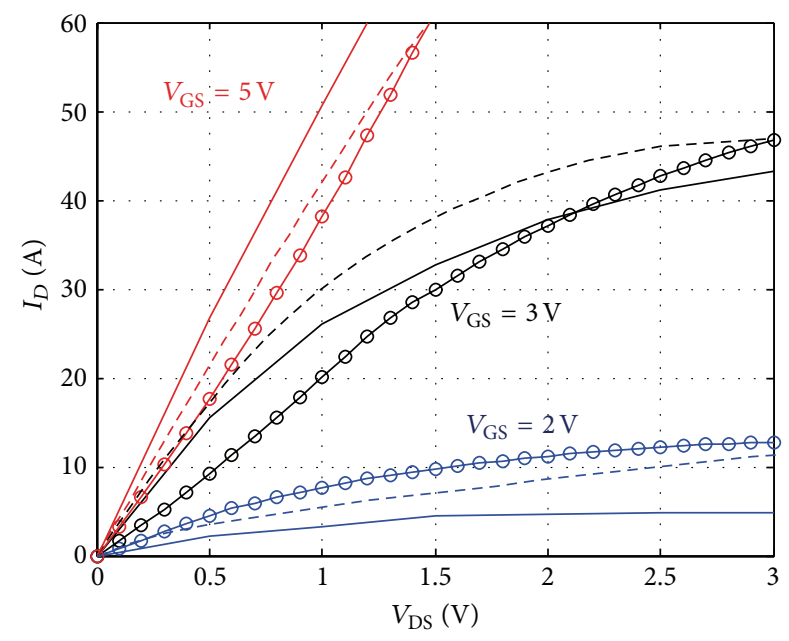

(c)

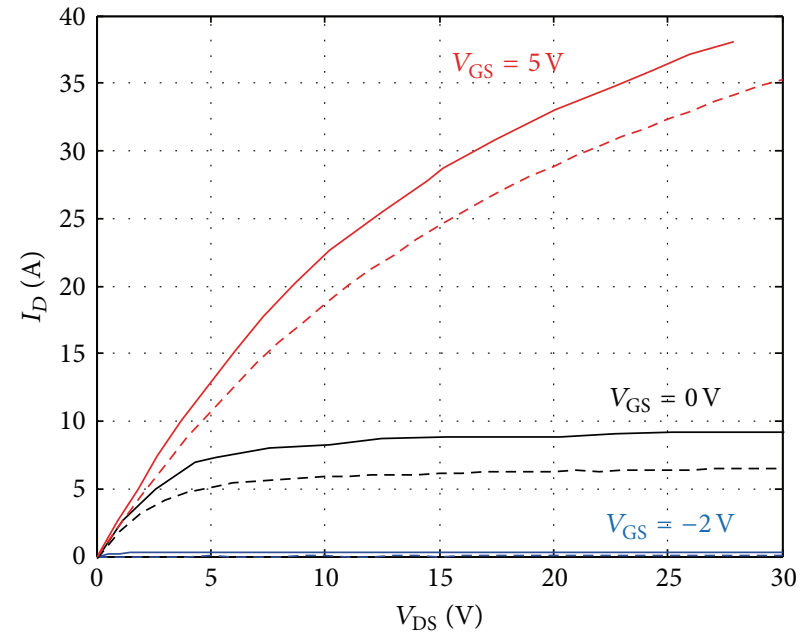

(b)

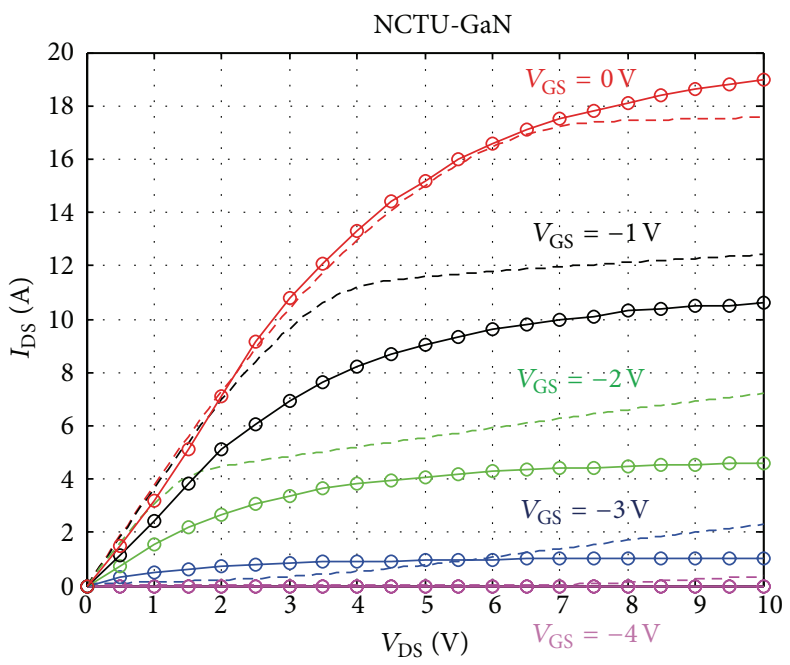

(d)

Figure 7: Output characteristics: (a) E-mode MOSFET, (b) D-mode MOSFET, (c) E-mode GaN FET, and (d) D-mode GaN FET.

$I_{\mathrm{DS}}(Y$-axis $)$ at a particular voltage $V_{\mathrm{DS}}(X$-axis) can be obtained from the simulated waveform. In addition to the on-resistance $R_{\mathrm{ON}}$ characteristics, the saturation voltage of the D-mode GaN FETs exhibits variance. The experimental results show that the average saturation voltage is at $V_{\mathrm{DS}}=$ $7 \mathrm{~V}$ and that the maximum saturation current is between 16 and $18 \mathrm{~A}$. The conduction resistance $R_{\mathrm{DS}(\mathrm{ON})}$ of the $\mathrm{D}$ mode GaN FET is $0.26 \Omega$, which is smaller than those of the two MOSFET power transistor $(0.32$ and $0.55 \Omega)$ but much larger than that of the E-mode GaN FET $(0.03 \Omega)$. In the future, $R_{\mathrm{DS}(\mathrm{ON})}$ of the $\mathrm{D}$-mode GaN FET can be improved using internal or external parallel methods $[6,7]$; therefore, uniform performance should be sorted.

3.2. Threshold Voltage. The measured threshold voltage $V_{\mathrm{TH}}$ is plotted in Figure 9. The conduction threshold current of the MOSFET power transistor is defined as $250 \mu \mathrm{A}$.
From the experimental results of the enhanced MOSFET, the threshold voltage $V_{\mathrm{TH}}$ is $3.21 \mathrm{~V}$, which is in the range specified in the datasheet $(2-4 \mathrm{~V})$. Furthermore, the conduction threshold current of the D-mode MOSFET is $250 \mu \mathrm{A}$; therefore, the gate threshold voltage $V_{\mathrm{TH}}$ is $-2.98 \mathrm{~V}$, which is in the range specified in the datasheet $(-4$ to $-2 \mathrm{~V})$. From the information in the manual, E-mode GaN FET conduction threshold current is defined as $3 \mathrm{~mA}$.

In Figure 9(a), when the output current of the E-mode GaN FET is $3000 \mu \mathrm{A}$, the gate threshold voltage $V_{\mathrm{TH}}$ is $1 \mathrm{~V}$, which is in the range specified in the datasheet $(0.7-2.5 \mathrm{~V})$. The output current $I_{D}$ of the D-mode GaN FET is plotted logarithmically in Figure 9(b). Near $V_{\mathrm{GS}}=-3.9 \mathrm{~V}$, the output current $I_{D}$ rises rapidly. Hence, this $V_{\mathrm{GS}}$ voltage is defined as the threshold voltage of the D-mode GaN FET. The threshold voltage $V_{\mathrm{TH}}$ of the E-mode GaN FET is much lower than that of the MOSFET. 


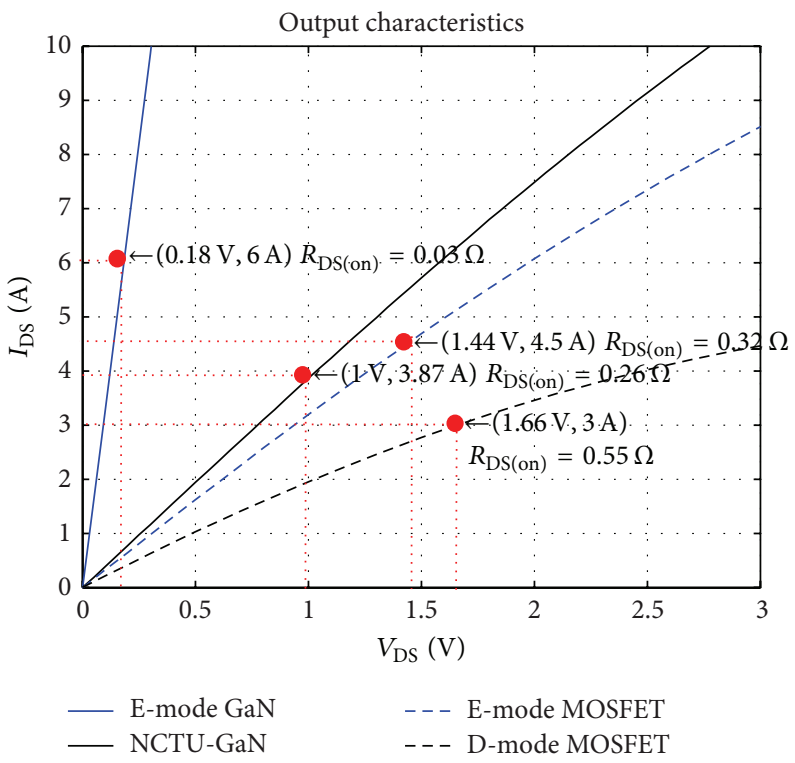

FIGURE 8: On-resistance of the four power transistors.

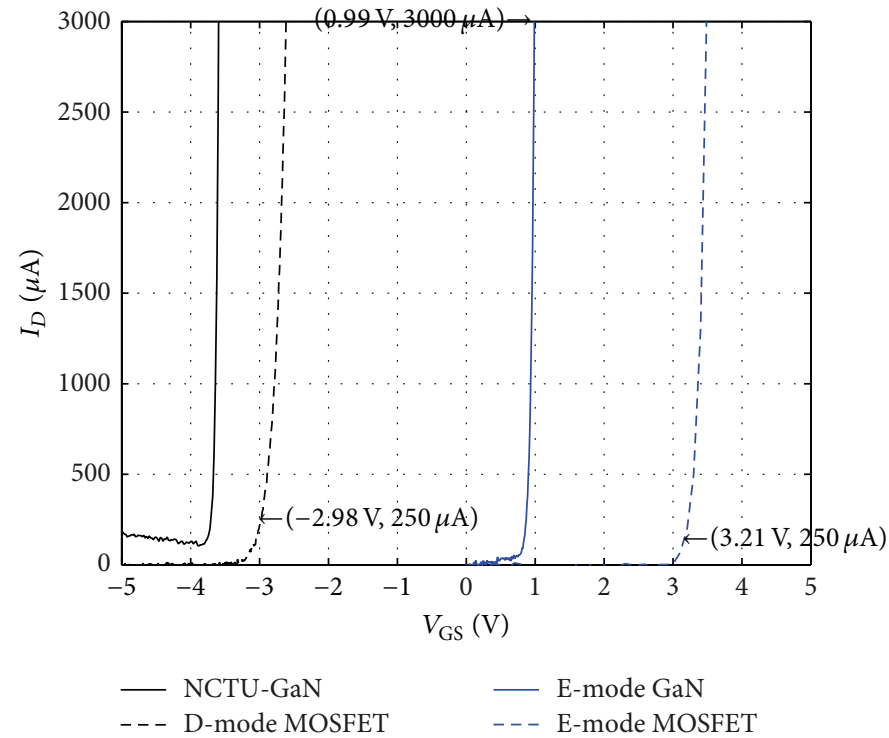

(a)

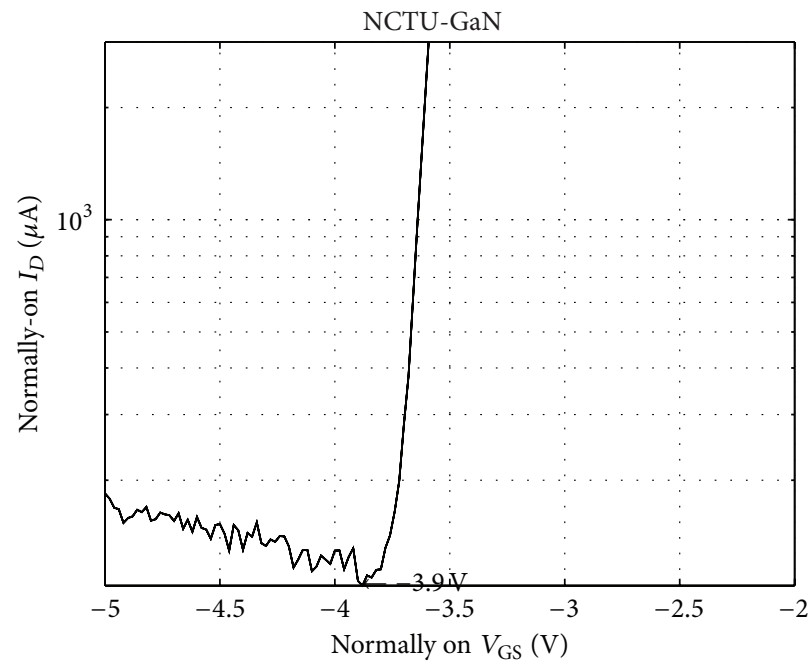

(b)

Figure 9: Transfer characteristics: (a) comparison of all transistors and (b) D-mode GaN FET.

3.3. Parasitic Capacitance. The datasheet provides power transistor parasitic capacitance characteristics, including the input capacitance $\left(C_{\text {ISS }}\right)$, output capacitance $\left(C_{\mathrm{OSS}}\right)$, and transpose capacitance $\left(C_{\mathrm{RSS}}=C_{\mathrm{GD}}\right)$, where $C_{\mathrm{ISS}}=C_{\mathrm{GS}}+$ $C_{\mathrm{GD}}, C_{\mathrm{OSS}}=C_{\mathrm{GD}}+C_{\mathrm{DS}}$, and $C_{\mathrm{RSS}}=C_{\mathrm{GD}}$. Figure 10 plots the measured parasitic capacitance values of the four power transistors. From Figure 10(a), for the E-mode MOSFET, at $V_{\mathrm{GS}}=0 \mathrm{~V}$ and the bias voltage $V_{\mathrm{DS}}=25 \mathrm{~V}$, the parasitic capacitances $C_{\mathrm{ISS}}, C_{\mathrm{OSS}}$, and $C_{\mathrm{RSS}}$ are $553.8,91.5$, and $27.3 \mathrm{pF}$, respectively, which are close to the datasheet values (540 (typ.), 90 (typ.), and 35 pF (typ.), resp.). From Figure 10(b), the E-mode GaN FET, at $V_{\mathrm{GS}}=0 \mathrm{~V}$, and a bias voltage $V_{\mathrm{DS}}=100 \mathrm{~V}$, parasitic capacitances $C_{\mathrm{ISS}}, C_{\mathrm{OSS}}$, and $C_{\mathrm{RSS}}$ are $473.7,301$, and $16.7 \mathrm{pF}$, respectively, which are close to the datasheet values (540 (max.), 350 (max.), and $12 \mathrm{pF}$ (max.)). From Figure 10(b), the D-mode MOSFET, at $V_{\mathrm{GS}}=-10 \mathrm{~V}$, 
TABLE 1: Comparison of datasheet and measured characteristics.

\begin{tabular}{|c|c|c|c|c|c|}
\hline Parameter & Symbol & Test conditions & $\begin{array}{c}\text { E-mode } \\
\text { GaN FET Value }\end{array}$ & $\begin{array}{c}\text { D-mode GaN } \\
\text { FET } \\
\text { Value } \\
\end{array}$ & Unit \\
\hline On-state resistance & $R_{\mathrm{DS}(\mathrm{ON})}$ & $\begin{array}{l}V_{\mathrm{GS}}=5 \mathrm{~V}, \\
V_{\mathrm{DS}}=0.18 \mathrm{~V}, I_{D}=6 \mathrm{~A} \\
V_{\mathrm{GS}}=0 \mathrm{~V}, \\
V_{\mathrm{DS}}=1 \mathrm{~V}, \\
I_{D}=3.87 \mathrm{~A}\end{array}$ & $\sim 25 \mathrm{~m}(\max .) / 30 \mathrm{~m}$ & $\begin{array}{c}- \\
-/ 0.26 \\
\end{array}$ & $\Omega$ \\
\hline $\begin{array}{l}\text { Gate threshold } \\
\text { voltage }\end{array}$ & $V_{\mathrm{TH}}$ & $\begin{array}{l}V_{\mathrm{GS}}=V_{\mathrm{DS}} \\
I_{D}=3 \mathrm{~mA} \\
V_{\mathrm{GS}}=7 \mathrm{~V} \\
\log \left(I_{D}\right)=\min . \mathrm{A}\end{array}$ & $\begin{array}{c}0.99 \\
-\end{array}$ & $\begin{array}{l}- \\
-3.9 \\
\end{array}$ & $\mathrm{~V}$ \\
\hline Input capacitance & $C_{\text {ISS }}$ & $\begin{array}{l}V_{\mathrm{GS}}=0 \mathrm{~V}, \\
V_{\mathrm{DS}}=100 \mathrm{~V}, \\
\text { osc. level }=30 \mathrm{mV} \\
(\mathrm{E}-\text { mode }) \\
V_{\mathrm{GS}}=-5 \mathrm{~V}, \\
V_{\mathrm{DS}}=50 \mathrm{~V}, \\
\text { osc. level }=30 \mathrm{mV} \\
\text { (D-mode) }\end{array}$ & $\begin{array}{c}540(\max .) / 473.7 \\
-\end{array}$ & $-\overline{172.7}$ & $\mathrm{pF}$ \\
\hline Output capacitance & $C_{\mathrm{OSS}}$ & $\begin{array}{l}V_{\mathrm{GS}}=0 \mathrm{~V}, \\
V_{\mathrm{DS}}=100 \mathrm{~V}, \\
\text { osc. level }=30 \mathrm{mV} \\
(\mathrm{E}-\text { mode }) \\
V_{\mathrm{GS}}=-5 \mathrm{~V}, \\
V_{\mathrm{DS}}=50 \mathrm{~V}, \\
\text { osc. level }=30 \mathrm{mV} \\
\text { (D-mode) }\end{array}$ & $\begin{array}{c}350(\max .) / 301 \\
-\end{array}$ & -164.4 & $\mathrm{pF}$ \\
\hline $\begin{array}{l}\text { Reverse transfer } \\
\text { capacitance }\end{array}$ & $C_{\mathrm{RSS}}$ & $\begin{array}{l}V_{\mathrm{GS}}=0 \mathrm{~V} \\
V_{\mathrm{DS}}=100 \mathrm{~V} \\
\text { osc. level }=30 \mathrm{mV} \\
(\mathrm{E}-\text { mode }) \\
V_{\mathrm{GS}}=-5 \mathrm{~V} \\
V_{\mathrm{DS}}=50 \mathrm{~V} \\
\text { osc. level }=30 \mathrm{mV} \\
\text { (D-mode) }\end{array}$ & $\begin{array}{c}12(\max .) / 16.7 \\
-\end{array}$ & $-\overline{-}$ & $\mathrm{pF}$ \\
\hline & & Before slash: datasheet & ash: measured & & \\
\hline
\end{tabular}

and a bias voltage $V_{\mathrm{DS}}=25 \mathrm{~V}$, parasitic capacitances $C_{\mathrm{ISS}}$, $C_{\text {OSS }}$, and $C_{\mathrm{RSS}}$ are $2361.7,243.7$, and $61.7 \mathrm{pF}$, respectively, and $C_{\text {ISS }}$ is under the value specified in the datasheet $(2800 \mathrm{pF}$ (typ.)), whereas $C_{\mathrm{OSS}}$ and $C_{\mathrm{RSS}}$ are close to the datasheet values (255 (typ.), $64 \mathrm{pF}$ (typ.)). For the E-mode GaN FET, at $V_{\mathrm{GS}}=0 \mathrm{~V}$ and $V_{\mathrm{DS}}=100 \mathrm{~V}$, parasitic capacitances $C_{\mathrm{ISS}}, C_{\mathrm{OSS}}$, $C_{\mathrm{RSS}}$ are $473.7,301$, and $16.7 \mathrm{pF}$, respectively, which are close to the datasheet values (540 (max.), 350 (max.), and $12 \mathrm{pF}$ $(\max )$.$) . For the D-mode GaN FET, at V_{\mathrm{GS}}=-5 \mathrm{~V}$ and $V_{\mathrm{DS}}=$ $50 \mathrm{~V}$, the parasitic capacitances $C_{\mathrm{ISS}}, C_{\mathrm{OSS}}$, and $C_{\mathrm{RSS}}$ are 72.7 , 64.4 , and $10.2 \mathrm{pF}$, respectively. Comparison of datasheet and measured characteristics are listed in Table 1, and Table 2 lists the important $I-V$ and capacitance model parameters for Eand D-mode used in this study.

According to (6a) and (6b), the parameters listed in Table 2 are used. The relationships $C_{\mathrm{ISS}}=C_{\mathrm{GS}}+C_{\mathrm{GD}}, C_{\mathrm{OSS}}=$ $C_{\mathrm{GD}}+C_{\mathrm{DS}}$, and $C_{\mathrm{RSS}}=C_{\mathrm{GD}}$ are used. Plots of $C_{\mathrm{ISS}}, C_{\mathrm{OSS}}$, and $C_{\mathrm{RSS}}$ are shown in Figures $10(\mathrm{c})$ and $10(\mathrm{~d})$. The simulated and experimental curves are similar.
3.4. Isolated Gate Drive Detection. A turn-off voltage of $0 \mathrm{~V}$ is used for the E-mode GaN FET; therefore, the full-conduction voltage is limited to $5.5 \mathrm{~V}$. For the D-mode GaN FET, the used turn-off voltage is $-5 \mathrm{~V}$, and full-conduction voltage is limited to $2 \mathrm{~V}$. Hence, the driving voltage for the E-mode GaN FET gate-to-source voltage is set to $0-5 \mathrm{~V}$; in other words, $V_{\text {Iso }}$ is set to $5 \mathrm{~V}$, and the D-mode GaN FET gate source driving voltage is set to -5 to $0 \mathrm{~V}$. At driving voltages of $0-5 \mathrm{~V}$ and -5 to $0 \mathrm{~V}$, the E- and D-mode MOSFET waveforms can be contrasted. Regardless of the MOSFET mode, the voltage probe was used to measure the voltage between the gate terminal and the ground terminal. The E-mode MOSFET waveforms are the same as the ideal isolated gate drive circuit detection signal, as depicted in Figures 3 and 4; the gate voltage when turned on is $+29 \mathrm{~V}$ and decreases to $0 \mathrm{~V}$ when turned off (Figure 11(a)). The D-mode MOSFET gate voltage waveform is $+24 \mathrm{~V}$, which decreases to 0 when turned off (Figure 11(b)). When measuring the E-mode GaN FET, the gate voltage is $+29 \mathrm{~V}$ when turned on, but a difference in 
TABLE 2: Simulation model parameters.

\begin{tabular}{|c|c|c|c|}
\hline \multirow{2}{*}{ Symbol } & \multicolumn{3}{|c|}{ GaN FETs model parameter } \\
\hline & Parameter & E-mode & D-mode \\
\hline$K_{P}$ & Transconductance parameter $\left(\mathrm{A} / \mathrm{V}^{2}\right)$ & 24 & 2.1 \\
\hline$V_{\mathrm{TO}}$ & Zero-bias threshold voltage & $1 \mathrm{~V}$ & $-3.9 \mathrm{~V}$ \\
\hline$\delta$ & Fitting parameter to adjust the curvature & 0.4 & 0.6 \\
\hline$C_{\mathrm{GS}}$ & External gate to source capacitance $(\mathrm{nF})$ & 0.45 & 0.06 \\
\hline \multirow{4}{*}{$C_{\mathrm{GD}}$} & External gate to drain capacitance & & \\
\hline & $C_{\mathrm{GD} 0}(\mathrm{nF})$ & 0.1 & 0.02 \\
\hline & $V_{J}(\mathrm{~V})$ & 0.66 & 0.75 \\
\hline & $m$ & 0.39 & 0.14 \\
\hline \multirow{4}{*}{$C_{\mathrm{DS}}$} & External drain to source capacitance & & \\
\hline & $C_{\mathrm{DS} 0}(\mathrm{nF})$ & 0.89 & 0.07 \\
\hline & $V_{J}(\mathrm{~V})$ & 0.4 & 0.35 \\
\hline & $m$ & 0.22 & 0.05 \\
\hline$C_{\text {stray }}$ & Stray capacitance in the circuit $(\mathrm{nF})$ & 2 & 2 \\
\hline$R_{L}$ & Voltage probe resistance $(\Omega)$ & $10 \mathrm{M}$ & $1 \mathrm{M}$ \\
\hline$R_{\mathrm{DS}(\text { off) }}$ & GaN FET turn-off resistance $(\Omega)$ & $7.647 \mathrm{M}$ & $904.8 \mathrm{k}$ \\
\hline
\end{tabular}

voltage level exists between the gate and the ground. The large change in the voltage level is in the $0-24 \mathrm{~V}$ range, as shown in Figure 12(a). D-mode GaN FETs exhibit the same phenomenon, as shown in Figure 12(b). The differences are caused by the turn-off impedance $R_{\mathrm{DS} \text { (off) }}$. The larger the turnoff impedance is, the smaller the leakage current is; the across voltage $V_{S \text { (off) }}$ is small, and the difference between sourceto-ground voltage value is close to 0 . Conversely, when the turn-off impedance is small, the leakage current is large, and the source-to-ground voltage approaches $+24 \mathrm{~V}$. Therefore, the turn-off ability of GaN FETs can indirectly screen device uniformity. Moreover, the impedance value can be quantified.

When $V_{\mathrm{GS}}=5 \mathrm{~V}$, the E-mode GaN FET turns on. The gate-to-ground voltage is $+29 \mathrm{~V}$; when $V_{\mathrm{GS}}=0 \mathrm{~V}$, the Emode GaN FET turns off, which is equivalent to the turnoff resistance $R_{\mathrm{DS} \text { (off); }}$; the voltage probe resistance is $R_{L}$. When the Kirchhoff circuit laws are applied, the power supply voltage $V_{\mathrm{DD}}$ through $R_{\mathrm{DS} \text { (off) }}$ and $R_{L}$ generate the leakage drain current $I_{\mathrm{DSS}}$. Through the isolated gate drive circuit architecture, the voltage probe resistance $R_{L}$ is $10 \mathrm{M} \Omega$ and power supply voltage $V_{\mathrm{DD}}$ is $24 \mathrm{~V}$. The $V_{S \text { (off) }}$ values for the two modes are $13.6 \mathrm{~V}$ and $23.0 \mathrm{~V}$, as shown in Figure $12(\mathrm{a})$. Substituting these values into (2), $R_{\mathrm{DS}(\text { off) }}$ is obtained as 7.647 and $0.435 \mathrm{M} \Omega$. Using a digital multimeter in series with the source terminal and the voltage probes $\left(R_{L}\right)$ to measure the GaN FET device during the turn-off state, the leakage currents $I_{\mathrm{DSS}}$ are obtained as 1.340 and $2.365 \mu \mathrm{A}$. By substituting $V_{S \text { (off) }}$ in (1a), leakage currents $I_{\text {DSS }}$ are obtained as 1.36 and $2.30 \mu \mathrm{A}$, which are similar to the measured values.

Next, the gate-to-ground voltage waveform of the Dmode GaN FET is measured. When $V_{\mathrm{GS}}$ is $0 \mathrm{~V}$, the $\mathrm{D}$-mode GaN FET drain and source conducts and shorts, and the source terminal voltage is $+24 \mathrm{~V}$. Because $V_{\mathrm{GS}}=0 \mathrm{~V}$, the gate terminal voltage is $+24 \mathrm{~V}$; when $V_{\mathrm{GS}}=-5 \mathrm{~V}$, the GaN FET is off, because the resistance of the D-mode GaN FET $R_{\mathrm{DS} \text { (off) }}$ is not large enough; therefore, leakage current flows, and the source terminal voltage relative to ground cannot be reduced to $0 \mathrm{~V}$. Next, the turn-off voltage of the D-mode GaN FET is measured using the $10 \mathrm{M} \Omega$ voltage probe; the value is always $19 \mathrm{~V}$. The turn-off impedance $R_{\mathrm{DS} \text { (off) }}$ is much lower than $10 \mathrm{M} \Omega$; therefore, the voltage probe is adjusted to $1 \mathrm{M} \Omega$ to repeat the experiments. $V_{S \text { (off) }}$ is in the $0-19 \mathrm{~V}$ range. $R_{\mathrm{DS} \text { (off) }}$ and voltage probe $R_{L}=1 \mathrm{M} \Omega$ divide the voltage, assuming that the probe is measured as $V_{S(\text { off })}$ voltage. From (1a), (1b), and (2), the leakage current $I_{\mathrm{DSS}}$ and turn-off impedance $R_{\mathrm{DS} \text { (off) }}$ can be obtained.

The waveform variability of the D-mode GaN FET is similar to that of the E-mode GaN FET, as shown in Figure 12(b). The D-mode GaN FET under a voltage probe $R_{L}=1 \mathrm{M} \Omega$ varies in the $16-20 \mathrm{~V}$ range. From (2), $R_{\mathrm{DS} \text { (off) }}$ of the D-mode GaN FET is $463.4 \mathrm{k} \Omega$ when $V_{S(\text { off })}$ is $16.4 \mathrm{~V}$ and $904.8 \mathrm{k} \Omega$ when $V_{S(\mathrm{off})}$ is $12.6 \mathrm{~V}$. However, when $V_{S(\text { off }}$ exceeds $24-5=19 \mathrm{~V}$, the turn-off impedance is insufficient and the transistor does not turn off.

In the E-mode GaN FET, the threshold voltage $V_{\mathrm{TH}}$ of the output characteristic curve $I-V$ model parameter is set to $1 \mathrm{~V}$ and $K_{P}$ is set to 24. An external capacitor is used as listed in Table 2 . In the isolated gate driver circuit architecture, the voltage probe resistance $R_{L}=10 \mathrm{M} \Omega$, power supply voltage $V_{\mathrm{DD}}=24 \mathrm{~V}$, and measuring voltage $V_{S \text { (off) }}=$ $13.6 \mathrm{~V}$. From (2), $R_{\mathrm{DS}(\mathrm{off})}$ impedance is derived as $7.647 \mathrm{M} \Omega$ when $V_{S(\text { off })}$ is $13.6 \mathrm{~V}$; therefore, when E-mode GaN FET turns off, the drain-to-source $R_{\mathrm{DS} \text { (off) }}$ is equivalent to a $7.647 \mathrm{M} \Omega$ resistor. $R_{L}$ is the internal voltage probe resistance, which is $10 \mathrm{M} \Omega$ at $10 \mathrm{x}$ magnification. In the D-mode GaN FET, the threshold voltage $V_{\mathrm{TH}}$ of its $I-V$ output characteristic curve model is $-3.9 \mathrm{~V}$ and $K_{P}$ is 2.1 . The external capacitor is used as parasitic capacitance. The turn-off impedance $R_{\mathrm{DS}(\mathrm{off})}$ of 


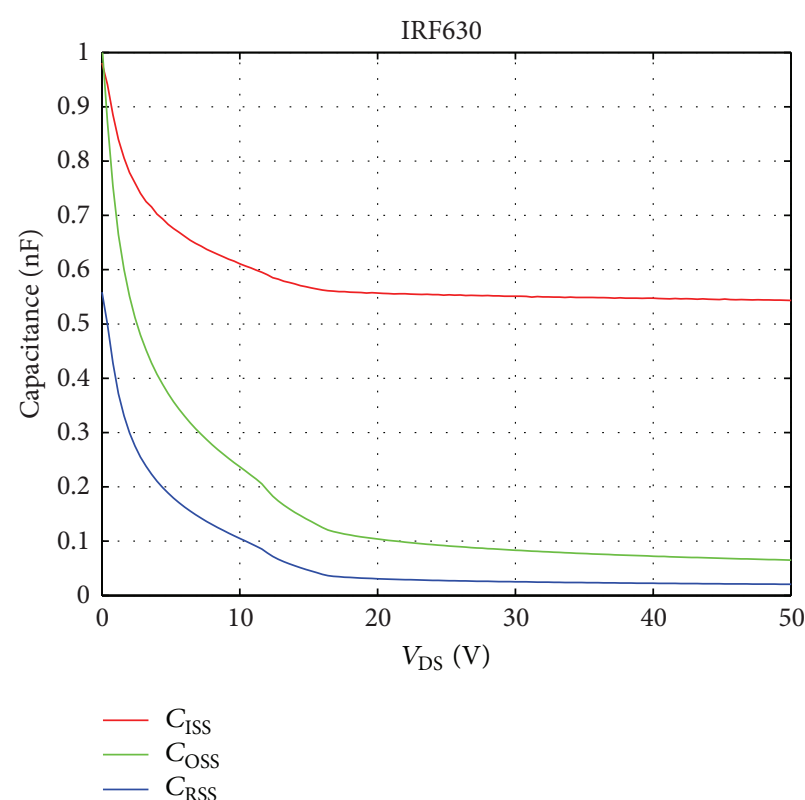

(a)

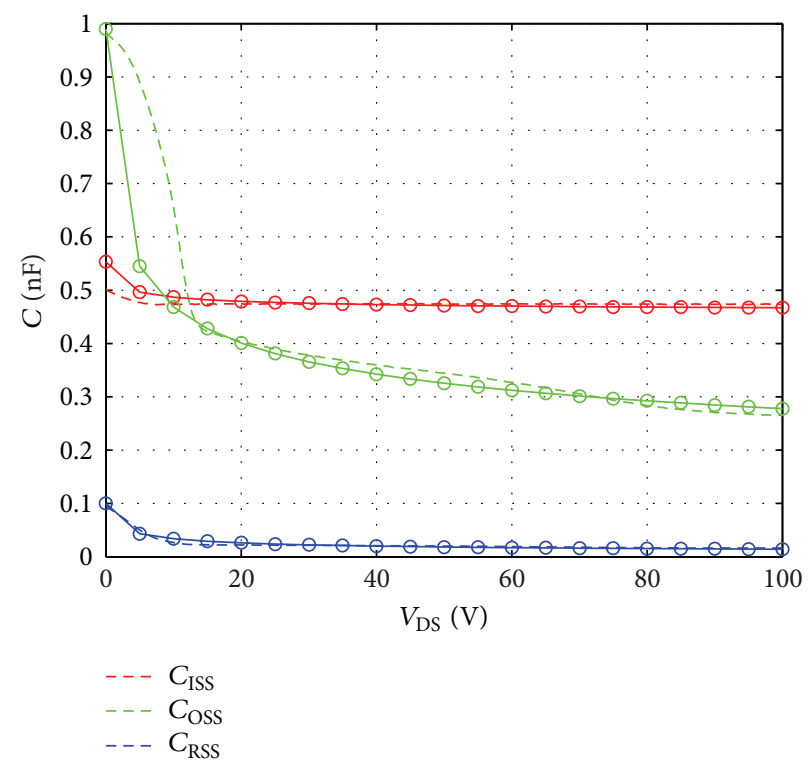

(c)

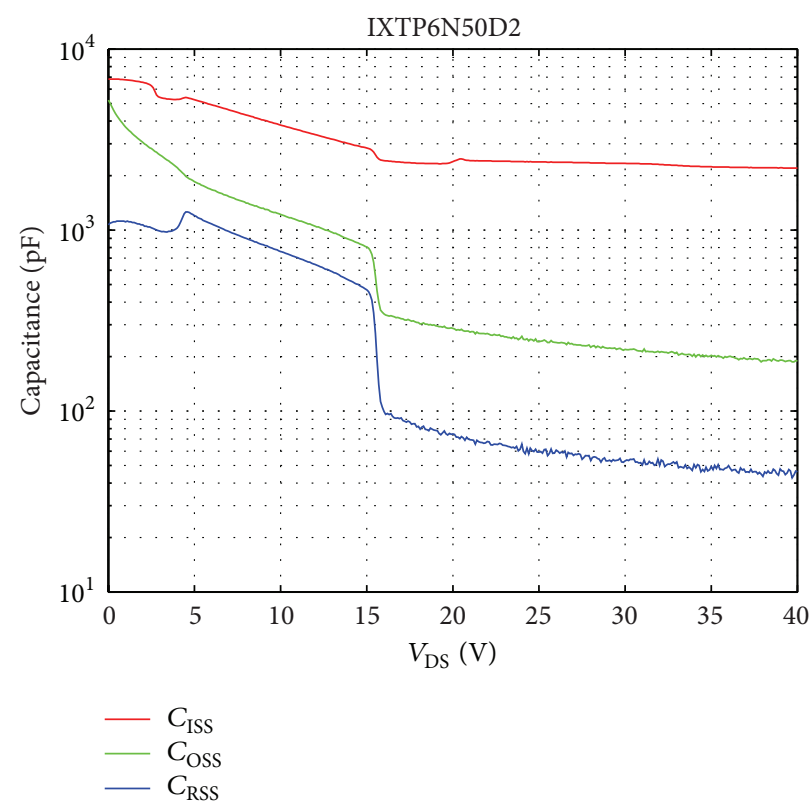

(b)

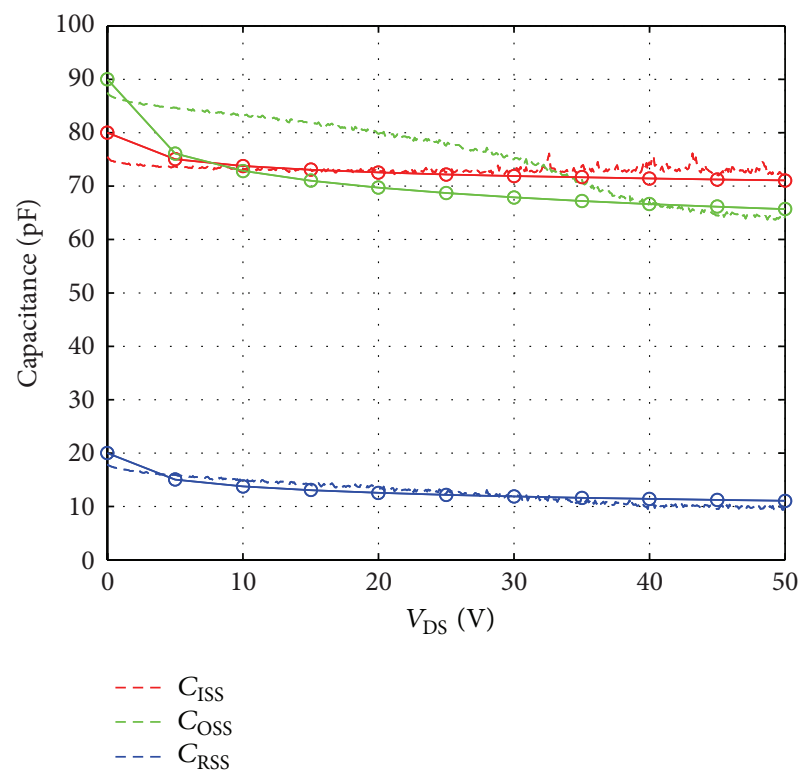

(d)

Figure 10: Parasitic capacitance: (a) E-mode MOSFET, (b) D-mode MOSFET, (c) E-mode GaN FET, and (d) D-mode GaN FET.

the experimental device using the 10x magnification voltage probe is much lower than $10 \mathrm{M} \Omega$. Hence, a $1 \mathrm{x}(1 \mathrm{M} \Omega)$ probe is used to measure $\left(R_{L}=1 \mathrm{M} \Omega\right)$; the power supply voltage $V_{\mathrm{DD}}=24 \mathrm{~V}$ and the measured $V_{S(\text { off })}$ voltage $=12.6 \mathrm{~V}$. From (2), when $V_{S(\text { off) }}$ voltage is $12.6 \mathrm{~V}, R_{\mathrm{DS} \text { (off) }}$ is $904.8 \mathrm{k} \Omega$. The equivalent turn-off resistance $R_{\mathrm{DS} \text { (off) }}$ between the drainsource is equivalent to $904.8 \mathrm{k} \Omega$.

SPICE circuits are established through the equivalent model described in Figure 5. The gate resistor uses $100 \Omega R_{G}$, and the E-mode GaN FET gate terminal wave signal $V_{\mathrm{GS}}$ voltage is $0-5 \mathrm{~V}$, whereas the D-mode GaN FET $V_{\mathrm{GS}}$ is -5 to $0 \mathrm{~V}$. Gate pulse width, period, and frequency of the PWM signal are $100 \mu \mathrm{s}, 500 \mu \mathrm{s}$, and $2 \mathrm{kHz}$, respectively. The numerical analysis software predicts that the gate drive circuit board has external drain-source stray capacitance $C_{\text {stray }}$ and that the actual measurements of waveform segments have a slower falling slope. Because of stray capacitance $C_{\text {stray }}$ parallel to drain-to-source and gate-to-drain, the turn-off $V_{\mathrm{GS}}$ slope falls slowly in the waveform. The estimates of the stray capacitance $C_{\text {stray }}$ value are $2 \mathrm{nF}$. The GaN FET gate detection simulation parameters are shown in Table 2.

Figures 13 and 14 present the E- and D-mode GaN FET gate detection simulation circuit waveform as shown in black line and measurement waveform as shown in orange 


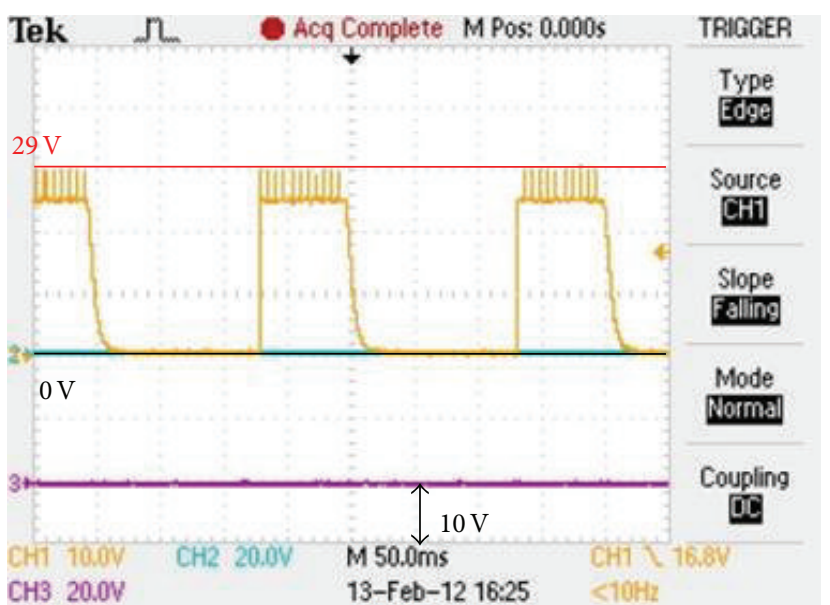

(a)

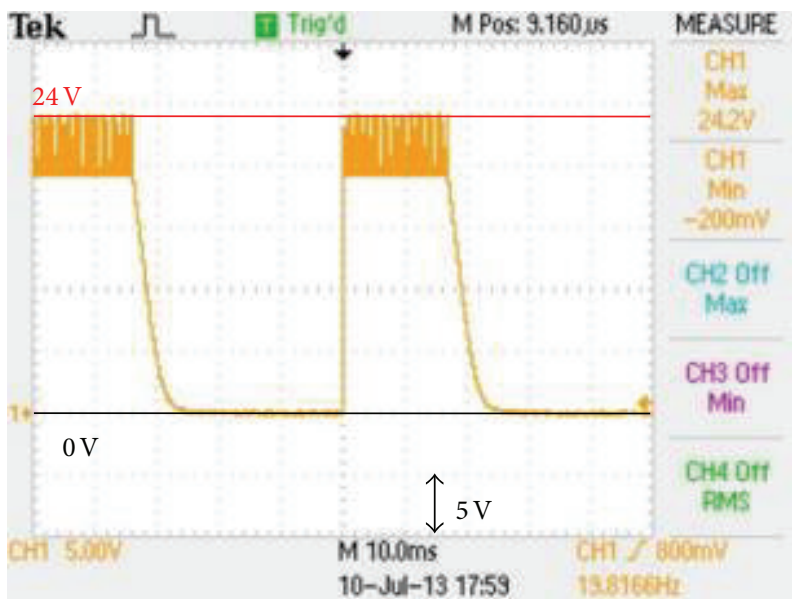

(b)

FIGURE 11: MOSFET gate drive detection signal waveforms: (a) E-mode and (b) D-mode.

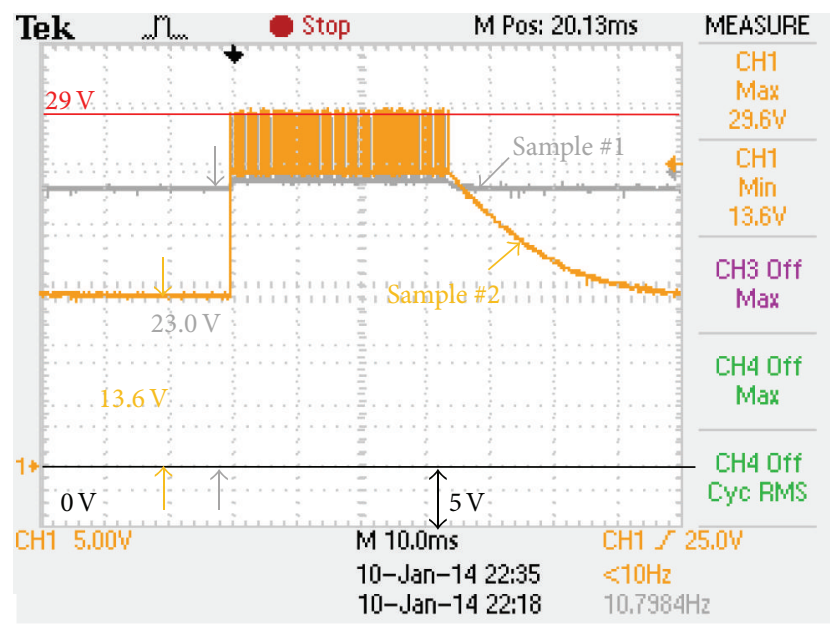

(a)

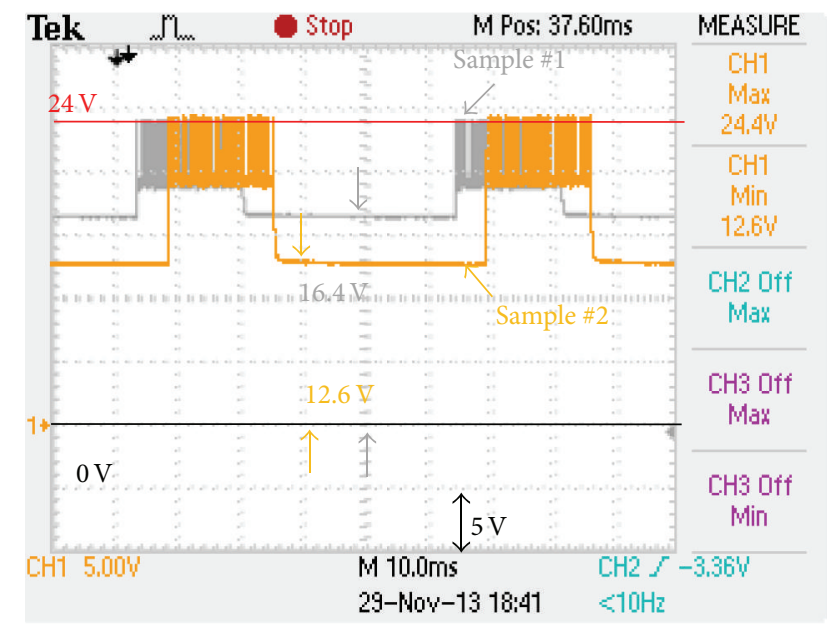

(b)

FIgURE 12: GaN FET gate drive detection signal waveforms: (a) E-mode and (b) D-mode.

line superimposing they are matching each other. When the isolated gate detection circuit stops sending the PWM signal to the gate terminal and GaN FET is set to close long enough, the falling slope waveform segment of the discharge through circuit stray capacitance $C_{\text {stray }}$ and load resistor $R_{L}$ from $+24 \mathrm{~V}$ floating voltage discharge to the voltage $V_{S \text { (off) }}$ is in accordance with the measurement. The influence of the parasitic capacitance of the input capacitance $C_{\mathrm{ISS}}$ on $V_{\mathrm{GS}}$ voltage switching waveform can be observed by enlarging the $V_{\mathrm{GS}}$ voltage signal timeline, as shown in the inset of Figures 13(a), 13(b) and 14(a), 14(b); the enlarged $V_{\mathrm{GS}}$ voltage signal charge and discharge waveforms are shown in Figures 13(c), 13(d), 14(c), and 14(d). The simulation and experimental gate voltage waveforms of the charge and discharge match perfectly.
The results of the screening and recording of the turn-off voltage $V_{S(\text { off })}$ and the corresponding $R_{\mathrm{DS} \text { (off) }}$ of the E-mode GaN FET voltages are shown in Figure 15. The off-resistance of E-mode GaN FETs is larger than $1 \mathrm{M} \Omega$, whereas those of Dmode GaN FETs are approximately in the $0.5-1.5 \mathrm{M} \Omega$ range.

\section{Conclusions}

The on-resistance $R_{\mathrm{DS}(\mathrm{ON})}$ of E-mode GaN FET and NCTU $\mathrm{D}$-mode GaN FET is $0.025-0.03 \Omega$ and $0.25-0.3 \Omega$; both of these values are lower than that of MOSFET. Nevertheless, NCTU's D-mode GaN FET can be further improved using the parallel method to reduce on-resistance. Regarding parasitic capacitance, the $C_{\mathrm{RSS}}$ of the E-mode GaN FET is far lower 


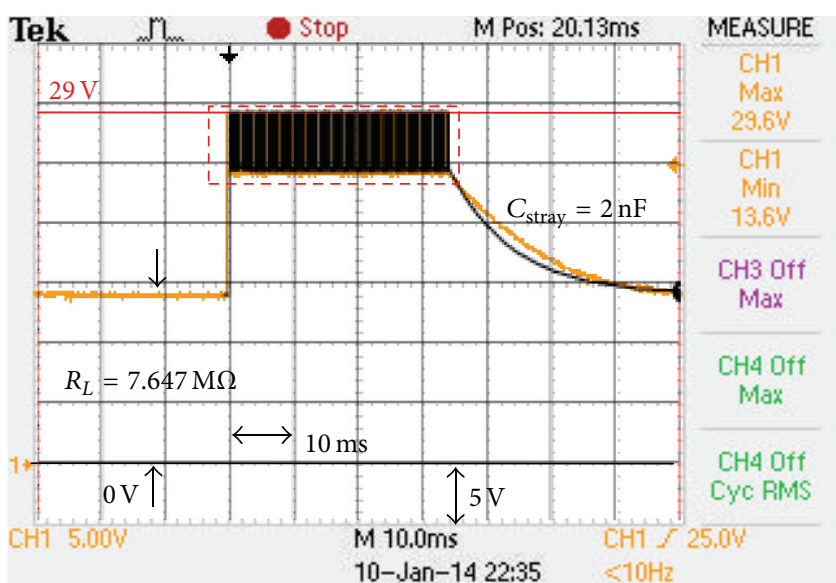

(a)

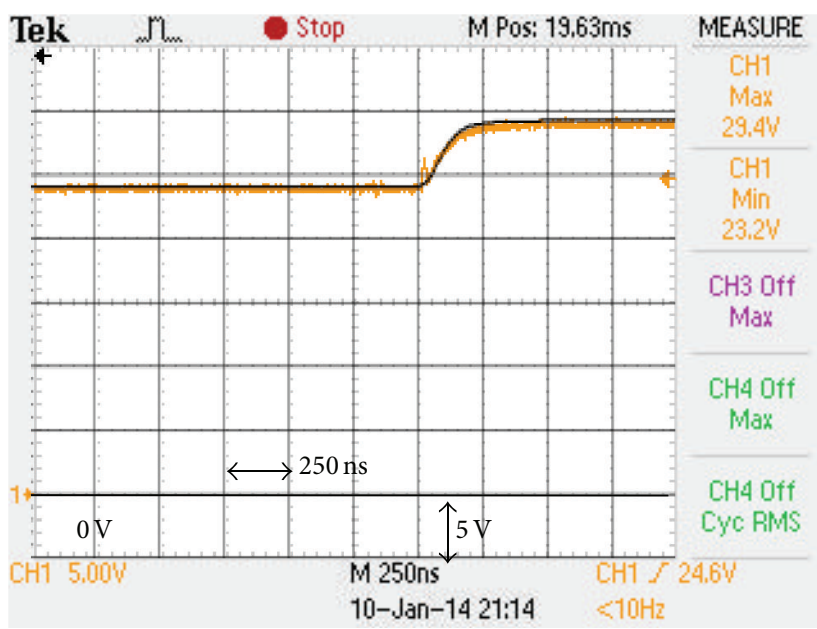

(c)

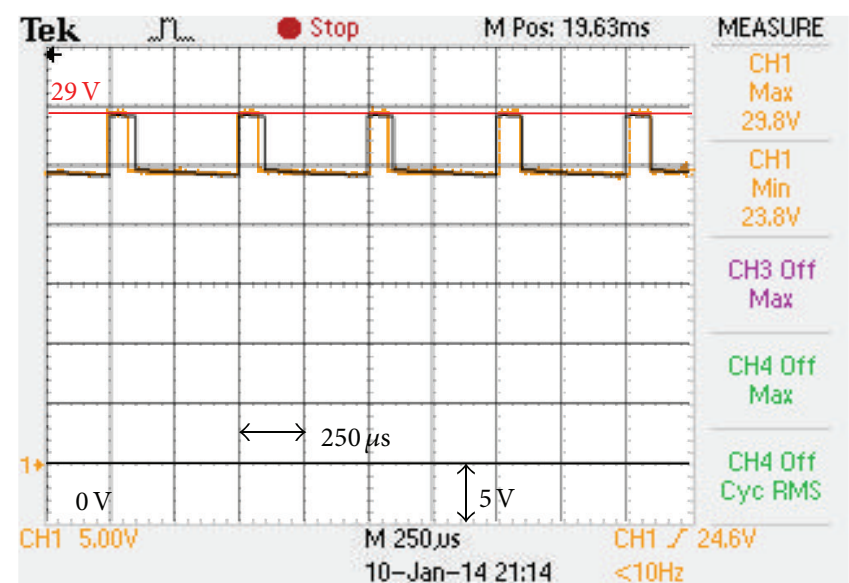

(b)

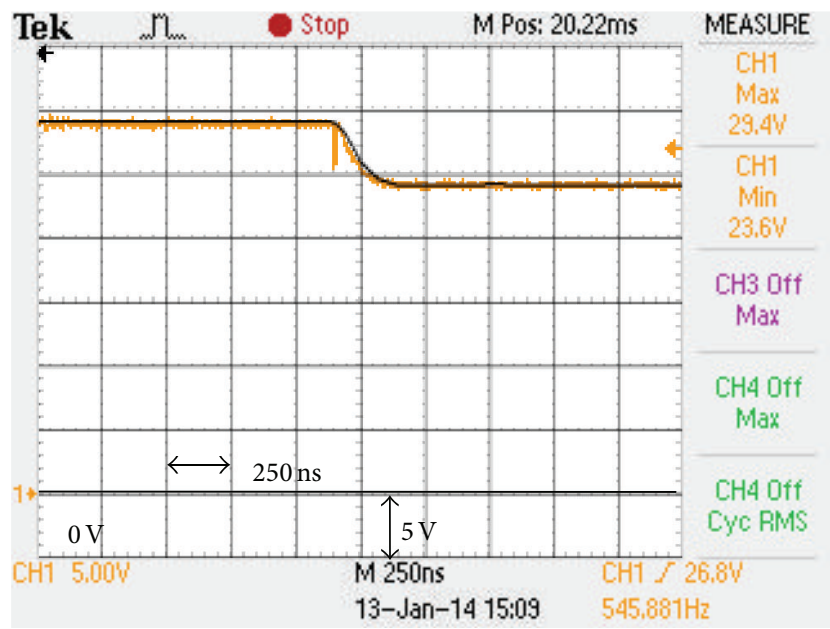

(d)

FIGURE 13: Simulation results of E-mode GaN FET.

than that of the enhanced MOSFET; a smaller $C_{\text {RSS }}$ capacitance value indicates that the Miller plain area is relatively short and that the switching time is shorter. Compared with the turn-off resistance of different samples, the electrical characteristics of each MOSFET device are highly consistent, whereas those of GaN FET exhibit less uniformity. GaN FETs are currently under development, and the electrical characteristics of each component are relatively unstable; the variability is larger than that in MOSFET. This study established a standardized electrical measurement procedure that provides necessary information for designers. In addition, a simple and accurate GaN FET model was established on the basis of the measured electrical characteristics. The simulation waveforms can be used to obtain information on GaN FET's internal parasitic capacitance, turn-off impedance $R_{\mathrm{DS} \text { (off) }}$, and stray capacitance $C_{\text {stray }}$ in the inverter circuit board. The proposed GaN FET isolated gate drive circuit screening method by $R_{\mathrm{DS} \text { (off) }}$ detection provides a simple uniformity sorting method. The results show that the higher the off-state voltage $V_{S \text { (off) }}$ is, the smaller the turn-off voltage
$V_{\mathrm{DS} \text { (off) }}$ is; in other words, the device has a lower $R_{\mathrm{DS} \text { (off) }}$. The leakage current in $\mathrm{GaN}$ devices is much larger than that in MOSFET devices in the turn-off state. The off-resistance of MOSFET is generally larger than $10 \mathrm{M} \Omega$. By contrast, the offresistance of E-mode GaN FETs is larger than $1 \mathrm{M} \Omega$, whereas those of D-mode GaN FETs are approximately in the 0.5$1.5 \mathrm{M} \Omega$ range. Devices with the same off-state voltage $V_{S(\text { off })}$ perform similarly. Moreover, the larger the turn-off resistance $R_{\mathrm{DS}(\text { off) }}$ is, the closer the characteristics are to those specified in the datasheet.

\section{Conflict of Interests}

The authors declare that there is no conflict of interests regarding the publication of this paper.

\section{Acknowledgments}

This work was supported by the CSIST Project CSIST0101-V108(104), Taiwan. The authors would like to thank 


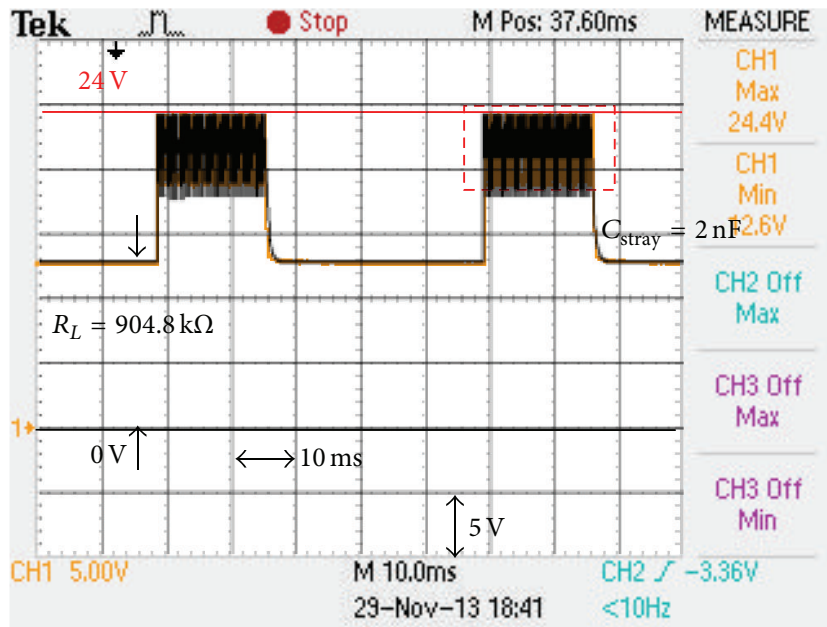

(a)

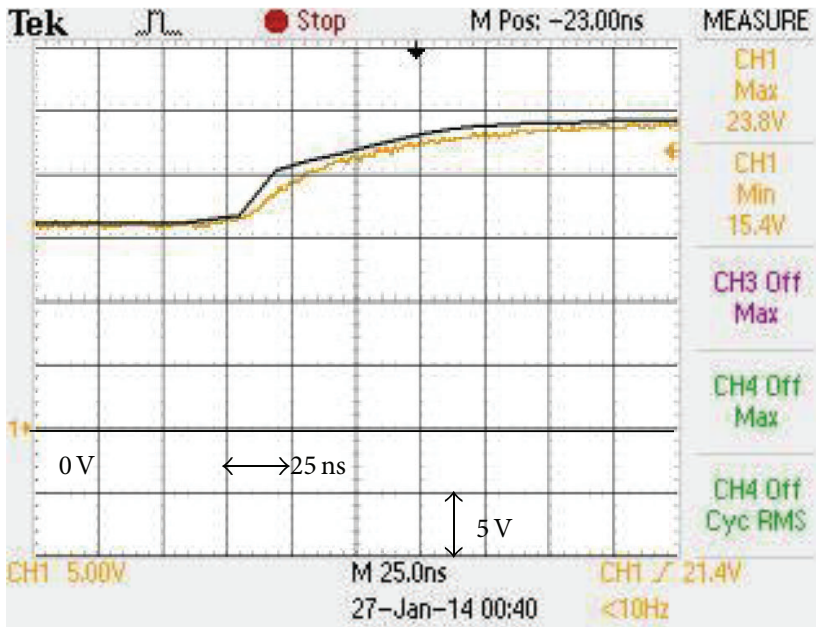

(c)

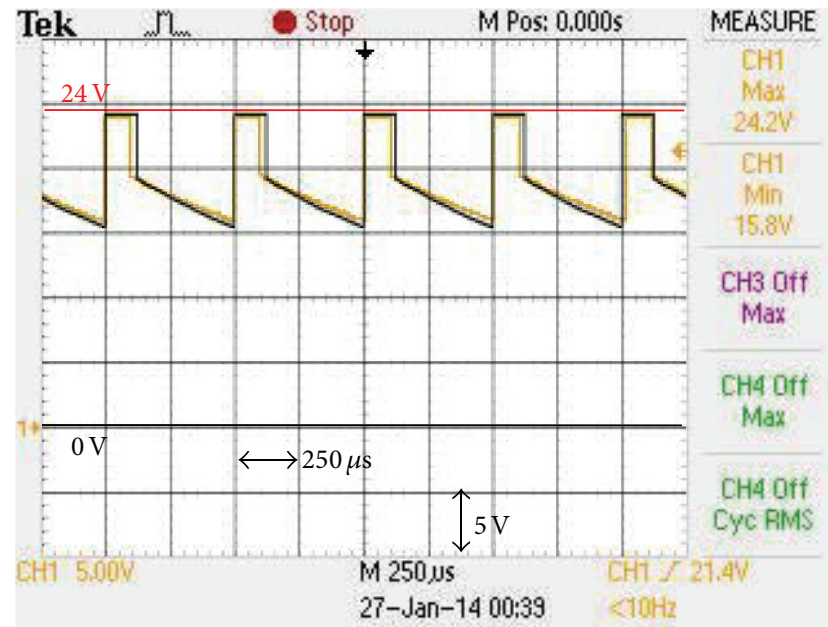

(b)

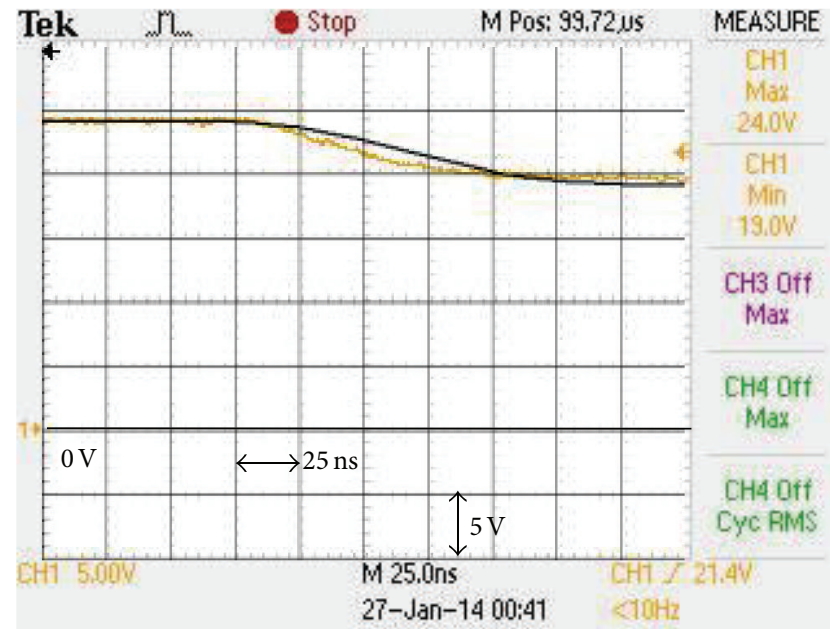

(d)

Figure 14: Simulation results of D-mode GaN FET.

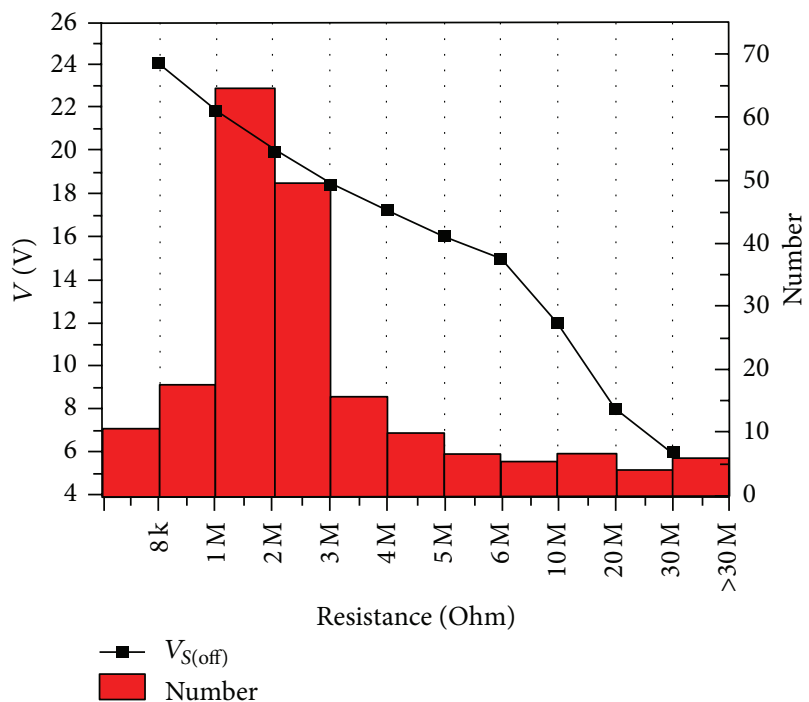

FIGURE 15: The turn-off voltage $V_{S(\text { off })}$ and the corresponding $R_{\mathrm{DS}(\text { off })}$ of E-mode GaN FETs. 
Professor Edward Yi Chang of NCTU for supporting GaN devices, Professor Stone Cheng of NCTU for supporting package technology, and National Nano Device Laboratories, Hsinchu, Taiwan, for their very helpful suggestions and technical support.

\section{References}

[1] B. Ozpineci, L. M. Tolbert, S. K. Islam, and M. Chinthavali, "Comparison of wide bandgap semiconductors for power applications," in Proceedings of the 10th European Conference on Power Electronics and Applications, Toulouse, France, September 2003.

[2] K. Shenai, M. Dudley, and R. F. Davis, "Rugged electrical power switching in semiconductors: a systems approach," Proceedings of the IEEE, vol. 102, no. 1, pp. 35-52, 2014.

[3] T. Ueda, M. Ishida, T. Tanaka, and D. Ueda, "GaN transistors on Si for switching and high-frequency applications," Japanese Journal of Applied Physics, vol. 53, no. 10, Article ID 100214, pp. $1-8,2014$.

[4] K. Shirabe, M. M. Swamy, J.-K. Kang et al., "Efficiency comparison between Si-IGBT-based drive and GaN-based drive," IEEE Transactions on Industry Applications, vol. 50, no. 1, pp. 566-572, 2014.

[5] B. Wang, N. Tipirneni, M. Riva, A. Monti, G. Simin, and a. E. Santi, "An efficient high-frequency drive circuit for GaN power HFETs," IEEE Transactions on Industry Applications, vol. 45, no. 2, pp. 843-853, 2009.

[6] P. Chou, S. Cheng, and S. Chen, "Evaluation of thermal performance of all-GaN power module in parallel operation," Applied Thermal Engineering, vol. 70, no. 1, pp. 593-599, 2014.

[7] S. Tsai, H. Hsu, Y. Tu, and E. Y. Chang, "Investigation of electrical and thermal properties of multiple AlGaN/GaN highelectron-mobility transistors flip-chip packaged in parallel for power electronics," Applied Physics Express, vol. 8, no. 3, Article ID 034101, 4 pages, 2015.

[8] H. Umegami, F. Hattori, Y. Nozaki, M. Yamamoto, and O. Machida, "A Novel high-efficiency gate drive circuit for normally off-type GaN FET,' IEEE Transactions on Industry Applications, vol. 50, no. 1, pp. 593-599, 2014.

[9] Y. Xi, M. Chen, K. Nielson, and R. Bell, "Optimization of the drive circuit for enhancement mode power GaN FETs in DCDC converters," in Proceedings of the 27th Annual IEEE Applied Power Electronics Conference and Exposition (APEC '12), pp. 2467-2471, IEEE, Orlando, Fla, USA, February 2012.

[10] J. Strydom and A. Lidow, "Driving eGaN transistors for maximum performance," 2011, http://epc-co.com/.

[11] P. Jacqmaer, J. Everts, R. Gelagaevz, P. Tant, and J. Driesen, "Fast robust gate-drivers with easily adjustable voltage ranges for driving normally-on wide-bandgap power transistors," in Proceedings of the 14th International Power Electronics and Motion Control Conference (EPE-PEMC '10), pp. T244-T251, Ohrid, Macedonia, September 2010.

[12] How to Drive GaN Enhancement Mode Power Switching Transistors, GaNSystems Application Note GN001, http://www .gansystems.com/.

[13] Designing Hard-switched Bridges with GaN, Transphorm Application Note AN0004, http://www.transphormusa.com/.

[14] L. Balogh, "Design and application guide for high speed MOSFET gate drive circuit," in Proceedings of the 2001 Power Supply Design Seminar, Texas Instruments, 2001.
[15] Design and Application Guide of Bootstrap Circuit for High Voltage Gate Drive IC, Fairchild Application Note AN-6076, http://www.fairchildsemi.com/.

[16] IRF630 ST Microelectronics Datasheet, http://www.st.com/.

[17] IXTA6N50D2, IXTP6N50D2, IXTH6N50D2 Datasheet, http:// ixdev.ixys.com/DataSheet/DS100177B\%28IXTA-TP-TH6N50D2\% 29.pdf.

[18] EPC2010 Datasheet, http://epc-co.com/.

[19] EPC GaN Transistor Parametric Characterization Guide, http:// epc-co.com.

[20] A. Ortiz-Conde, F. J. García Sánchez, J. J. Liou, A. Cerdeira, M. Estrada, and Y. Yue, "A review of recent MOSFET threshold voltage extraction methods," Microelectronics Reliability, vol. 42, no. 4-5, pp. 583-596, 2002.

[21] Agilent Technologies, Agilent B1505A Power Device Analyzer/Curve Tracer, Step by Step Measurement Handbook for Power MOSFET, http://www.agilent.com/.

[22] Fairchild AN-9005, Driving and Layout Design for Fast Switching. Super-Junction MOSFETs, http://www.semi.com/ an/AN/AN-9005.pdf.

[23] K. Shah and K. Shenai, "Simple and accurate circuit simulation model for gallium nitride power transistors," IEEE Transactions on Electron Devices, vol. 59, no. 10, pp. 2735-2741, 2012.

[24] Y. Cheng, M.-C. Jeng, Z. Liu et al., "A physical and scalable I-V model in BSIM3v3 for analog/digital circuit simulation," IEEE Transactions on Electron Devices, vol. 44, no. 2, pp. 277-287, 1997.

[25] IsSpice4 User's Guide, http://www.intusoft.com. 

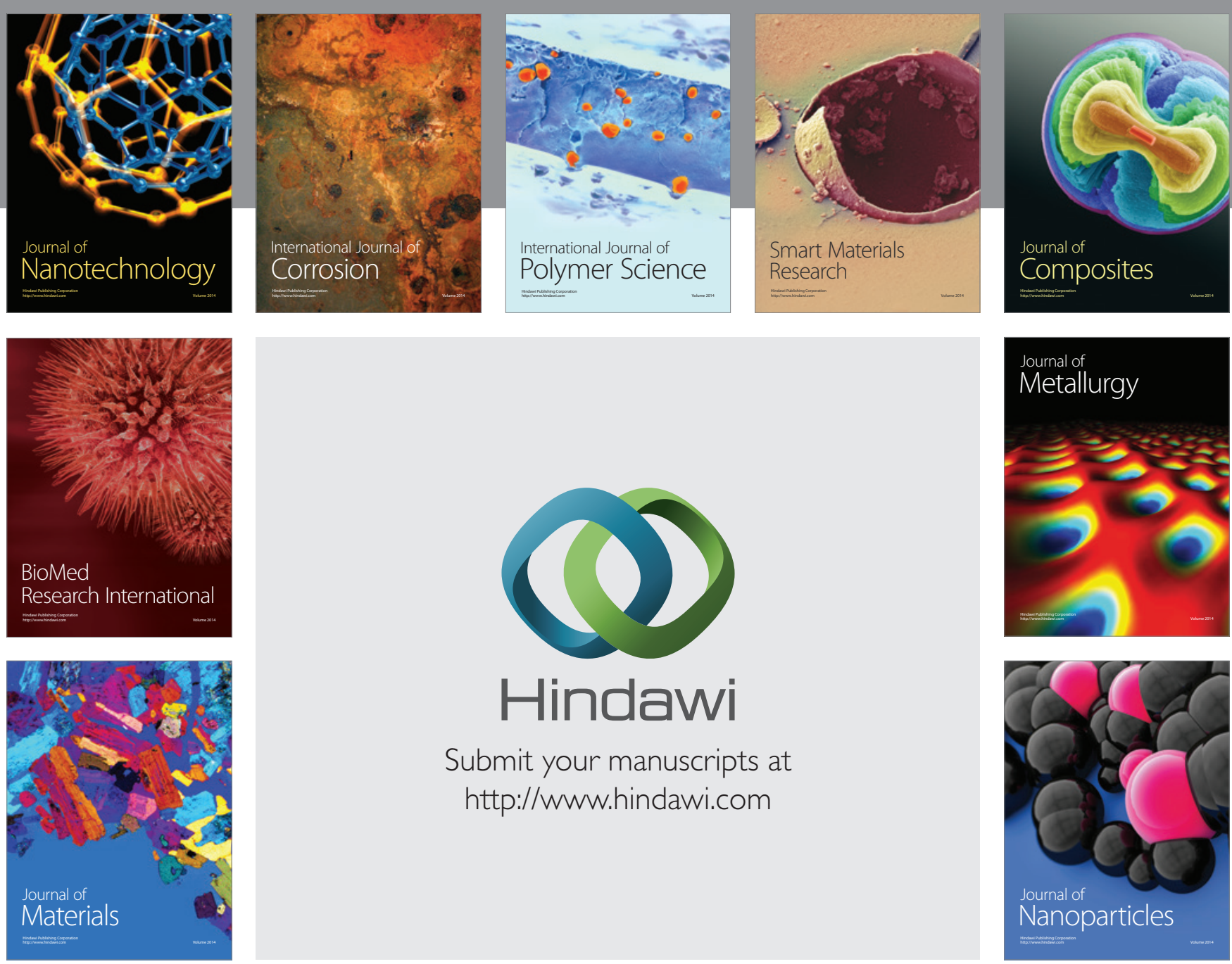

Submit your manuscripts at http://www.hindawi.com
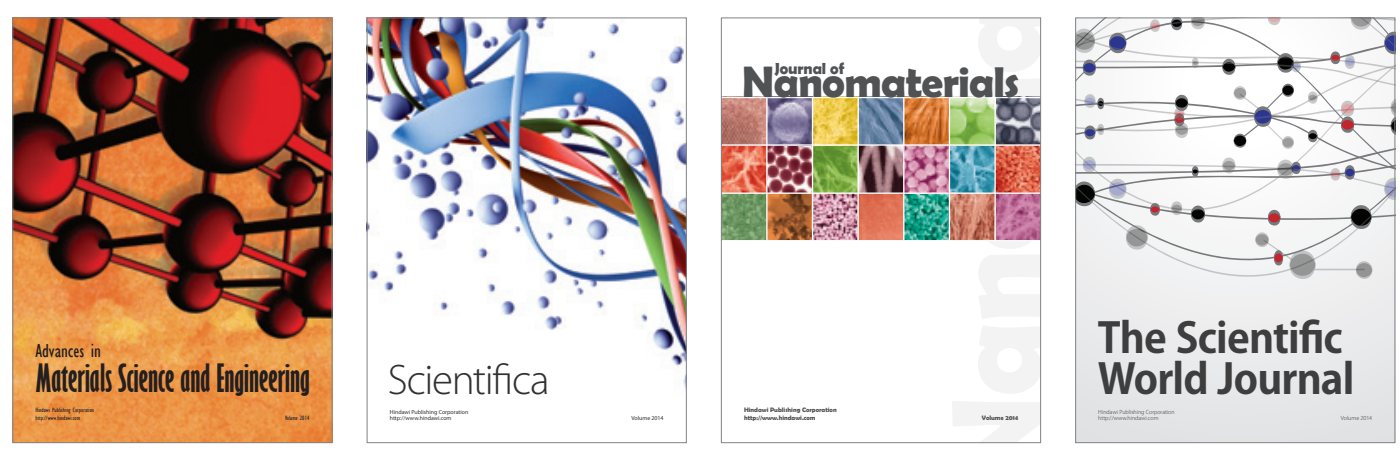

\section{The Scientific World Journal}
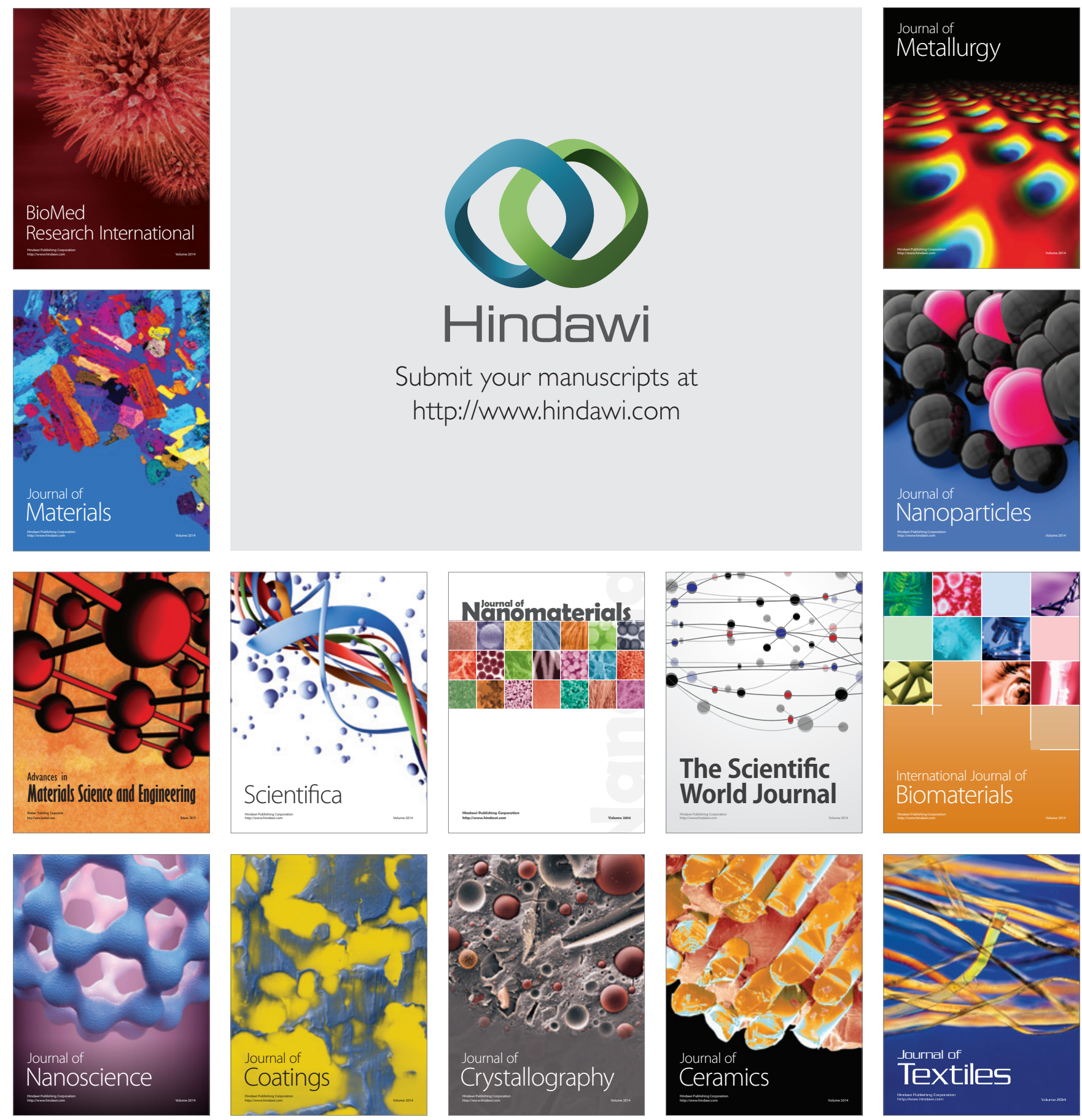